\title{
Emergent Potts Order in a Coupled Hexatic-Nematic XY model
}

\author{
Victor Drouin-Touchette $\odot,{ }^{1}$ Peter P. Orth $\odot,{ }^{2,3, *}$ Piers Coleman $\odot,{ }^{1,4}$ Premala Chandra $\odot,{ }^{1}$ and Tom C. Lubensky $\odot^{5}$ \\ ${ }^{1}$ Center for Materials Theory, Rutgers University, Piscataway, New Jersey 08854, USA \\ ${ }^{2}$ Ames Laboratory, Ames, Iowa 50011, USA \\ ${ }^{3}$ Department of Physics and Astronomy, Iowa State University, Ames, Iowa 50011, USA \\ ${ }^{4}$ Department of Physics, Royal Holloway, \\ University of London, Egham, Surrey TW20 OEX, United Kingdom \\ ${ }^{5}$ Department of Physics and Astronomy, University of Pennsylvania, \\ 209 South 33rd Street, Philadelphia, Pennsylvania 19104, USA
}

(Received 25 March 2021; revised 19 November 2021; accepted 4 January 2022; published 7 March 2022)

\begin{abstract}
Many two-dimensional physical systems ranging from atomic-molecular condensates to lowdimensional superconductors and liquid-crystal films are described by coupled XY models. The interplay of topology and competing interactions in these XY systems drives new kinds of emergent behavior relevant in both quantum and classical settings. Such coupled U(1) systems further introduce rich physics, bringing topology into contact with fractionalization and deconfinement. Motivated by a hidden-order phase transition in isotropic liquid-crystal $54 \mathrm{COOBC}$ films, we study the finite-temperature phase diagram of a minimalist hexatic-nematic XY model. We identify a small region of composite Potts order above the vortex-binding transition; this phase is characterized by relative hexatic-nematic ordering though both variables are disordered. We propose that the Potts order results from a confinement of fractional vortices into extended nematic defects and discuss the broader implications of fractional vortices and composite ordering in the wider class of coupled XY condensates.
\end{abstract}

DOI: $10.1103 /$ PhysRevX.12.011043

Subject Areas: Condensed Matter Physics,

Statistical Physics

\section{INTRODUCTION}

Two-dimensional XY models have played a central role in the understanding of topology in statistical and condensed matter physics. These U(1) fluids undergo a Kosterlitz-Thouless phase transition [1-3], where the binding of topological defects leads to a phase with algebraic long-range order. Two-dimensional melting, magnetism, and superfluidity are among the many settings for this universal phenomenon.

The interaction of distinct condensates described by coupled XY models $[4,5]$ is realized in many physical systems including atom-molecular mixtures in BoseEinstein condensates [6,7], multicomponent superconductors $[8,9]$, twisted bilayer graphene [10], and nematic or smectic liquid crystals [11-13]. The coupling of U(1) systems further introduces rich physics, as the constraints between allowed topological defects can lead to fractionalized excitations [14-16].

" porth@iastate.edu

Published by the American Physical Society under the terms of the Creative Commons Attribution 4.0 International license. Further distribution of this work must maintain attribution to the author(s) and the published article's title, journal citation, and DOI.
Liquid crystals, with their fluid structure and micron-sized defects, have played an important role in realizing and characterizing Kosterlitz-Thouless (KT) physics. Furthermore, the related development of novel crystalline and orientational orders seen in these systems, such as nematic and sliding phases [17], have been widely generalized to other areas, including cold atoms [18,19], frustrated magnetism [20-22], quantum Hall stripe phases [23-25], and other strongly correlated electron systems [26-29]. Borrowing concepts from these latter areas, we are thus motivated to address an unexplained observation in liquid crystals; we hope that our study can provide insights into emergent ordering processes of interacting $\mathrm{U}(1)$ condensates.

The observation of a sharp specific heat signal in the multistep melting sequence [30-32] of certain freestanding liquid-crystal films remains an outstanding mystery. Such transitions are typically associated with the binding of topological defects $[1,2,33]$ without acute thermodynamic signatures. Furthermore, the enigmatic discrete transition occurs in the phase with free vortices where no long-range order is expected. Here we revisit this unsolved problem, bringing to it modern concepts of confinement, vortex fractionalization, and composite ordering, while also taking advantage of the increased computational power now available.

We study a minimalist coupled hexatic-nematic XY model $[11,12,34,35]$ that captures the relevant degrees of 
freedom of the experimental system and is consistent with its symmetries. The elementary excitations of this system are hexatic and nematic vortices, which carry different charge associated with their phase winding. It is known that integer-charge vortices can split into multiple fractional charge vortices linked by domain-wall strings [6] if it is energetically favored. Here we explore whether such a vortex fractionalization can lead to an emergent three-state Potts phase above a KT binding temperature, where elementary vortices form charge-neutral pairs. Using large-scale parallel-tempering Monte Carlo simulations, we identify a small region where there is a sharp transition leading to Potts order even though the underlying hexatic and nematic angles remain disordered; quasi-long-range order (QLRO) in these variables develops at a lower temperature via a KT transition. This emergent composite order, in which disordered nematic and hexatic angles order relative to each other, is a new member in the rich class of vestigial orders that has been identified as playing a key role in the phase diagrams of frustrated magnets [20-22,36-45], unconventional superconductors [28,29,46-53], ultracold atoms [54], and liquid crystals [55]. Our numerical findings are supported with analytical arguments that describe how fractionalization of nematic vortices leads to extended vortex cores and a separation of the two transitions. The sequence of upper Potts and lower KT transitions provides a natural explanation of the observed multistep melting process in certain liquid crystals and might also be observable in other physical settings such as atom-molecular mixtures.

Let us now discuss the organization of this paper. In Sec. II, we provide an summary of our main results, also presenting the experimental liquid-crystal setting that motivates our work. Next in Sec. III, using analytic methods, we explore the thermodynamic phase diagram of the coupled hexatic-nematic model as a function of the coupling, highlighting the interplay of vortex confinement, Potts domain walls, and fractionalization. In Sec. IV, we describe the Monte Carlo algorithm and the observables that we use to identify the different thermodynamic phases. In Sec. V, we present our Monte Carlo simulations, including the main result: the finite-temperature phase diagram of the coupled hexatic-nematic model. We discuss the behavior of the system in different regions of the phase diagram and end the paper in Sec. VI with concluding remarks and outlook for future research directions.

\section{SUMMARY OF THE MAIN RESULTS}

Here we study a specific generalized XY model whose two types of topological defects become highly constrained when the two phases lock together. This leads to the possibility of novel phases, depending on the type of defects which then develop. Our Hamiltonian, for which a schematic phase diagram is presented in Fig. 1, has the following form:

$$
\begin{aligned}
\mathcal{H}= & -J_{2} \sum_{\langle i, j\rangle} \cos \left[\left(\varphi_{i}-\varphi_{j}\right)\right]-J_{6} \sum_{\langle i, j\rangle} \cos \left[3\left(\vartheta_{i}-\vartheta_{j}\right)\right] \\
& -\lambda^{\prime} \sum_{i} \cos \left[3\left(\vartheta_{i}-\varphi_{i}\right)\right] .
\end{aligned}
$$

Here, $\langle i, j\rangle$ runs over nearest-neighbor sites of a $2 \mathrm{D}$ square lattice. The phase diagram of the uncoupled $\left(\lambda^{\prime}=0\right)$ model has nematic- and hexatic-KT transitions. Above these defect-binding temperatures, free vortices are present with "charge" $q$ associated with the phase winding $2 \pi q$ around them; here, $q_{\vartheta}=[(\Delta \vartheta) / 2 \pi]=\frac{1}{3}$ and $q_{\varphi}=[(\Delta \varphi) / 2 \pi]=1$.

When the coupling $\lambda^{\prime}$ is finite, the two vortex types are no longer independent because now the system tends to minimize the third term of Eq. (2.1). The phases consequently lock together so that $\vartheta-\varphi \equiv[(2 \pi) / 3] n$ $(\bmod 2 \pi)$ where $n$ is an integer (the $\bmod 2 \pi$ simply associates $\tilde{n}_{i}=-1,-2$ with $n_{i}=2,1$, respectively). There are therefore three inequivalent relative alignments of $\varphi$ and $\vartheta$ [Fig. 2(c)]. This suggests the presence of a welldefined three-state Potts order parameter, which we write as

$$
M_{\varsigma}=\sum_{i} \exp \left[i \varsigma_{i}\right]=\sum_{i} \exp \left[i\left(\vartheta_{i}-\varphi_{i}\right)\right]
$$

Since $\varsigma_{i}=[(2 \pi) / 3] n_{i}$ for finite $\lambda^{\prime}$, then a finite $m_{\varsigma}=$ $\left\langle\left|M_{\varsigma}\right|\right\rangle$ is the direct consequence of long-range order in the relative Potts variable.

An important consequence of a finite $\lambda^{\prime}$ is the relative locking of the hexatic and nematic fields. The vortex charges are now related by the expression

$$
q_{\vartheta} \equiv q_{\varphi}+\frac{\Delta n}{3}(\bmod 1), \quad\left(\lambda^{\prime} \neq 0\right) .
$$

Here, $\Delta n$ is the number of Potts domain walls that separate different domains of relative Potts ordering, i.e., $\varsigma \rightarrow$ $\varsigma+[(2 \pi) / 3]$. Even though $\vartheta$ and $\varphi$ are isotropic, the finite coupling $\lambda^{\prime}$ locks their relative orientation, imposing the constraint (2.3) that has two important consequences:

(i) $q_{\vartheta}$ defects are bound to Potts domain walls [Fig. 1(a), in black] which follows from Eq. (2.3) as $\frac{1}{3}=0+\frac{1}{3}$.

(ii) Integer $q_{\varphi}=1$ vortices are composites formed of $q_{\vartheta}=\frac{1}{3}$ defects bound by domain walls [Fig. 1(d)] since, referring to Eq. (2.3), $3 \times \frac{1}{3}=1+0$.

When there are no free $q_{9}=\frac{1}{3}$ charges and thus no "dangling" Potts domain walls, a unique local Potts order parameter can be defined. Therefore, the binding of $q_{\vartheta}=\frac{1}{3}$ vortices at the hexatic-KT transition for $J_{2} \ll J_{6}$ results in a network of Potts walls [Fig. 1(b)] separating distinct local Potts domains. At some lower temperature, one expects one such domain to dominate the system leading to long-range Potts order and a finite value of the order parameter $m_{\varsigma}$ from Eq. (2.2). Indeed, previous computational work in this 


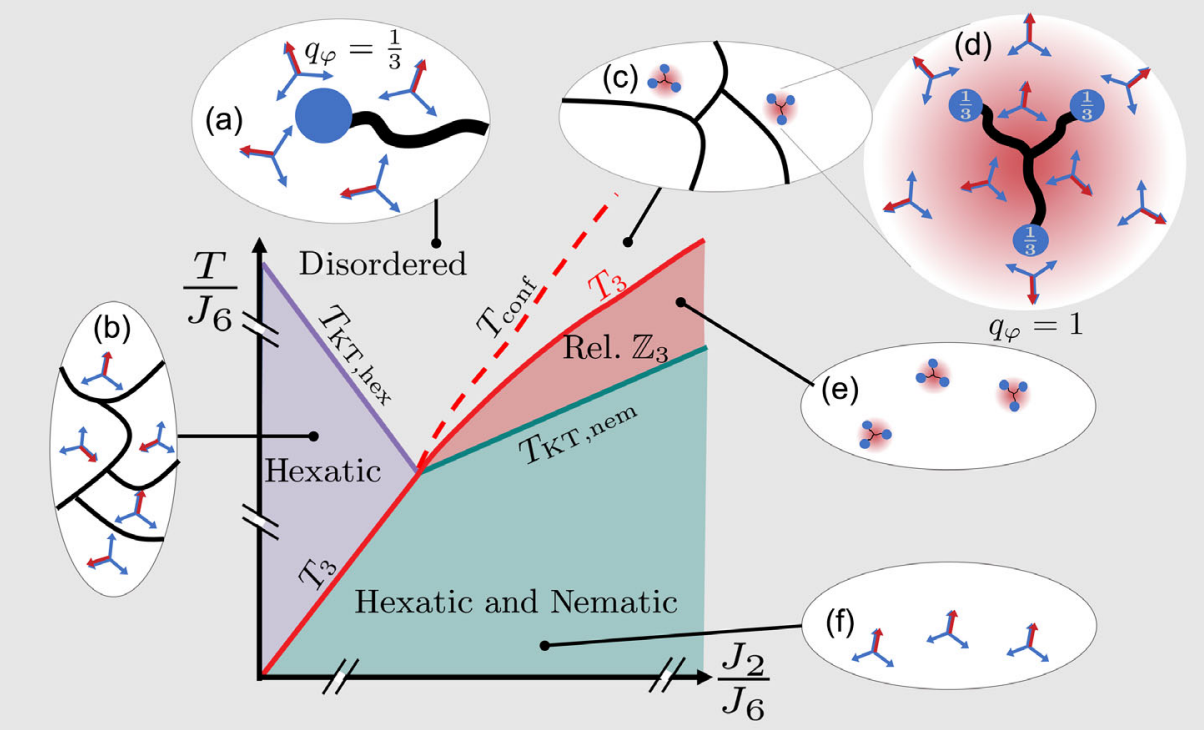

FIG. 1. Schematic phase diagram of the coupled XY model (2.1) (center); $T_{\mathrm{KT} \text {,hex }}$ and $T_{\mathrm{KT} \text {,nem }}$ are the binding temperatures of hexatic $q_{\vartheta}=1 / 3$ and nematic $q_{\varphi}=1$ vortices, respectively. These are depicted as blue triarrows for the hexatic $\vartheta$ variable and as red arrows for the nematic $\varphi$ variable. $T_{3}$ refers to a three-state Potts transition that is well established at temperatures below $T_{\mathrm{KT} \text {,hex }}$, and we find they also persist above $T_{\mathrm{KT} \text {,nem. }}$. The disordered phase is characterized by free hexatic vortices $\left[q_{\vartheta}=1 / 3\right.$; see blue dot in (a)], which correspond to fractional nematic vortices attached to a Potts domain wall (in black depicted on the nematic variable here) where $\Delta n= \pm 1$. For $J_{2} \ll J_{6}$, these hexatic vortices then bind in neutral pairs below $T_{\mathrm{KT} \text {, hex }}$. The hexatic phase is shown in (b). The presence of multiple Potts domains leads to disorder in $\varphi$ (red arrows). Below $T_{3}$ a single Potts domain covers the whole system, and the absence of free vortices leads to order in the hexatic and nematic variables, as shown in (f). At larger $J_{2} / J_{6}$, a new sequence of phase transition develops. First, at $T_{\text {conf }}$ the $q_{\vartheta}=1 / 3$ defects undergo a confinement into composite $q_{\varphi}=1$ vortices. In this confined phase (c), the absence of free $q_{\vartheta}=1 / 3$ defects and their associated "dangling" Potts domain walls leads to a network of local Potts domains. Within each domain, neither $\varphi$ nor $\vartheta$ is ordered, and composite nematic vortices exist. An enlargement of one such vortex is shown in (d): The composite carries charge $q_{\varphi}=1$ and $q_{\vartheta}=3 \times \frac{1}{3}=1$. The core contains a structure where three $q_{\vartheta}=1 / 3$ vortices are bound by adjoining Potts domain walls. Below $T_{3}$, the system develops a single Potts domain (e) containing free composite nematic vortices.

parameter regime confirmed the presence of a Potts ordered phase below the hexatic-KT transition [34,35] as indicated in the schematic phase diagram in Fig. 1.

The situation on the right side of the phase diagram $\left(J_{2} \gg J_{6}\right)$ is more subtle. From previous studies [6,56-64], we expect that there exists a parameter regime where bound states of three $q_{\vartheta}$ vortices form above the nematic-KT transition [Fig. 1(d)]. This is driven by the confinement of $q_{\vartheta}=1 / 3$ fractional vortices into a composite and extended $q_{\varphi}=1$ vortex. Confinement $[65,66]$ is well known from elementary particle physics, where it describes the binding of quarks into integer-charge baryons or mesons, but it also occurs frequently in condensed matter systems such as magnets [40,67-70], quantum Hall systems [71], coupled atomic-molecular superfluids [6], and generalized XY models with vector magnetic or nematic degrees of freedom [14,59,61].

In our model (2.1), local Potts order develops at this "confinement" temperature, where confinement of fractional nematic vortices drives the binding of three elementary $q_{\vartheta}=1 / 3$ hexatic vortices [see Fig. 1(e)]. This bound-state formation removes the dangling ends of Potts domain walls and thus enables the appearance of a well-defined Potts order parameter in the system. If this scale was merely a crossover, then there could not be any lower-temperature transition into Potts long-range order. This is because for a crossover, "dangling" domain walls could continue to exist on long length scales. By continuity with the $J_{2} \ll J_{6}$ side, the low-temperature ordered phase must have long-range order (LRO) in the relative Potts variable. Therefore, one has to embrace the results that the confinement is a true phase transition [61]. For temperatures lower than the confinement transition, the associated Potts domain walls disappear, and there is long-range Potts order [Fig. 1(e)], again reflected in a finite value of the order parameter $m_{\varsigma}$. This will certainly be the case when the composite vortices bind at $T_{\mathrm{KT} \text {,nem }}$, but the coincidence of these two transition temperatures would surely be indicative of an underlying unknown symmetry.

\section{A. Experimental motivation}

Optical reflectivity, electron diffraction, and specific heat measurements [Fig. 2(a)] in freestanding 2D films of 54COOBC (n-pentyl-4'-n-pentanoyloxybiphenyl-4carboxylate) [30-32] provide experimental motivation for our work. Theoretically, a two-stage melting sequence was expected in these $2 \mathrm{D}$ films with an intermediate hexatic phase 


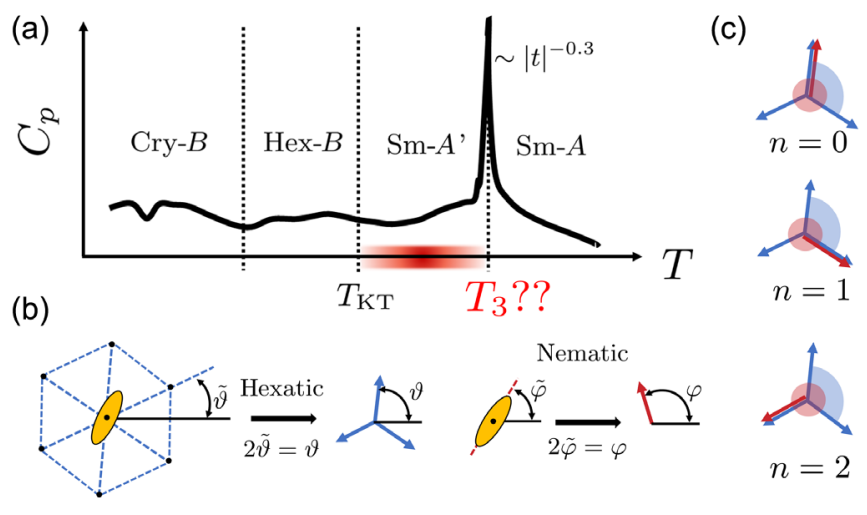

FIG. 2. (a) Schematic of the specific heat curve obtained by Chou et al. [32] upon melting of 54COOBC. (b) The hexatic bond-orientational $\vartheta$ and molecular nematic $\varphi$ degrees of freedom in the liquid-crystal film and their representations in the generalized XY model of Eq. (2.1). The relative orientation of neighboring nematic molecules is an open question, and we therefore do not represent it here. (c) Three relative configurations of $\varsigma=2 \pi n / 3=\vartheta-\varphi$ [as presented in Eq. (2.2)] for the angular variables in Eq. (2.1). Shaded areas represent the domain of their respective variables.

residing between the isotropic liquid and the $2 \mathrm{D}$ crystalline solid, as observed in several freestanding films [72,73]. This two-stage melting process can become a single first-order transition if the disclination core energy is lower than some critical value $[74,75]$.

By contrast, in 54COOBC experimentalists observed three-step melting with two phases separating the solid and the liquid states, a surprise since modifications to the Kosterlitz-Thouless-Halperin-Nelson-Young (KTHNY) theory cannot lead to a sequence of three transitions. In particular, they detected a mystery intermediate liquid phase with no long-range orientational order. With decreasing temperature, it is sandwiched between a (disordered) isotropic smectic- $A(\mathrm{Sm}-A)$ phase and a hexatic (Hex- $B)$ phase with bond orientational QLRO; the experimentalists referred to this unexpected "hidden order" $(\mathrm{HO})$ as the smectic- $A^{\prime}\left(\mathrm{Sm}-A^{\prime}\right)$ phase [Fig. 2(a)]. At even lower temperatures, the system develops positional QLRO in the hexagonal crystalline phase $(\mathrm{Cry}-B)$. The transition into the "mystery" phase is characterized by a pronounced specific heat anomaly; its scaling exponent was reported as $\alpha_{54 \text { СООвС }}=0.30 \pm 0.07$ [30], suggesting that the HO $\mathrm{Sm}-A^{\prime}$ phase has three-state Potts order at temperatures above the conventional vortex-binding transition. This is in striking disagreement with the broad features predicted by the 2D melting theory of KTHNY [1,2,33,76-79] at the hexatic to isotropic transition.

Theoretically, this problem can be studied using a minimalist coupled hexatic-nematic model on a 2D square lattice $[11,12,34,35]$, where the hexatic degrees of freedom $\left(\tilde{\vartheta}\right.$, invariant under $\tilde{\vartheta}_{i} \rightarrow \tilde{\vartheta}_{i}+2 \pi n / 6$ for integer $\left.n\right)$ describe the orientational order of the neighboring molecules' center of mass. A nematic degree of freedom ( $\tilde{\varphi}$, invariant under $\tilde{\varphi}_{i} \rightarrow \tilde{\varphi}_{i}+\pi n$ for integer $n$ ) is included, as this compound is a member of a large family of molecules presenting nematicinduced behaviors. This term is thought to arise from the interplay of the orientation of the rod-shaped molecules of $54 \mathrm{COOBC}$ with respect to a fixed laboratory axis [Fig. 2(b)] with the other neighboring molecules. Proposed types of nematic-induced orders in liquid crystals are the herringbone state $[80,81]$ and the nematic bond-orientational ordering [13,82-84], among others. In both cases, a nematic order emerges due to the in-plane rodlike shape of the molecules. Although there has been no observation of nematic order in 54COOBC $[30,31,85,86]$, we believe that such a term can generically appear there due to the microscopic nematic nature of the molecules, as in the $n m$-OBC family [87]. We do not attempt to give a detailed microscopic analysis of the origin of the nematic order, and we address some experimental and theoretical concerns in Sec. VI. The model then becomes

$$
\begin{aligned}
\mathcal{H}= & -J_{2} \sum_{\langle i, j\rangle} \cos \left[2\left(\tilde{\varphi}_{i}-\tilde{\varphi}_{j}\right)\right]-J_{6} \sum_{\langle i, j\rangle} \cos \left[6\left(\tilde{\vartheta}_{i}-\tilde{\vartheta}_{j}\right)\right] \\
& -\lambda^{\prime} \sum_{i} \cos \left[6\left(\tilde{\vartheta}_{i}-\tilde{\varphi}_{i}\right)\right] .
\end{aligned}
$$

The hexatic-nematic coupling $\lambda^{\prime}>0$ is the minimal symmetry-allowed coupling between the nematic and hexatic fields and favors a parallel relative alignment $[11,12,88]$. It could arise from van der Waals interactions between the molecules, favoring distinct alignments microscopically. By rescaling $\vartheta=2 \tilde{\vartheta}$ and $\varphi=2 \tilde{\varphi}$ so that the degrees of freedom are vectors rather than directors, we can reexpress Eq. (2.4) as the generalized XY model in Eq. (2.1).

The ensemble of measurements on $54 \mathrm{COOBC}$ thin films suggest Potts ordering at temperatures above that of the nematic vortex binding [30-32]. We therefore probe whether we can tune Eq. (2.1) to a parameter regime of its phase diagram where there is a Potts phase above the nematic Kosterlitz-Thouless transition. Using a combination of computational studies and analytic arguments, we find that it indeed exists (Fig. 1, on the right side), and that the separation of the two transitions is in reasonable agreement with experimental observation.

\section{HEXATIC-NEMATIC XY MODEL}

For technical reasons, in the rest of the paper we study a model in the same universality class as that of Eqs. (2.1) and (2.4). This equivalent model allows us to connect with previous numerical work $[34,35]$ that acts as a benchmark. This is done through a transformation of the hexatic and nematic degrees of freedom of the minimal coupled model of Eq. (2.4). Rescaling the angles $\theta_{i}=6 \tilde{\vartheta}_{i}, \phi_{i}=2 \tilde{\varphi}_{i}$ (equivalently, $\theta_{i}=3 \vartheta_{i}, \phi_{i}=\varphi_{i}$ ) so they both cover the range $\theta_{i}, \phi_{i} \in[0,2 \pi)$ yields the dimensionless expression 


$$
\begin{aligned}
\mathcal{H} / J= & -\Delta \sum_{\langle i, j\rangle} \cos \left(\phi_{i}-\phi_{j}\right)-(2-\Delta) \sum_{\langle i, j\rangle} \cos \left(\theta_{i}-\theta_{j}\right) \\
& -\lambda \sum_{i} \cos \left(\theta_{i}-3 \phi_{i}\right) .
\end{aligned}
$$

Here, we introduce $J=\frac{1}{2}\left(J_{2}+J_{6}\right), \lambda=\lambda^{\prime} / J$, and $\Delta \equiv$ $J_{2} / J$ such that $0 \leq \Delta \leq 2$ covers all exchange coupling ratios $J_{2} / J_{6}$. A value of $\Delta=1$ corresponds to the isotropic limit $J_{2}=J_{6}$. This description of our minimal model in terms of two $\mathrm{O}(2)$ variables is most useful for the Monte Carlo study we provide in the next sections, hence, the change of variables. Note that we interchangeably denote the symmetry of the underlying XY systems as $\mathrm{U}(1)$ or $\mathrm{O}(2)$, with the former corresponding to condensates, whereas the latter corresponds to liquid-crystal settings.

In the remainder of this section, we provide an intuitive and semianalytical description of the expected phase diagram of this model. Starting from the uncoupled model is Sec. III A, we first show that the hexatic-nematic coupling term is a relevant perturbation that tends to induce relative $\mathbb{Z}_{3}$ Potts order at temperatures larger than the KT transition temperature. Furthermore, we point out in Sec. III B the important role of vortex excitations in the system, especially as to how they differ from those in the uncoupled model. In Sec. III C, we show why the fractionalization of the nematic vortices leads to extended vortex cores and that this is a necessary requirement for the Potts transition to occur above the KT transition. Finally, we review some numerical results of related models in Sec. III D.

\section{A. Uncoupled model and relevance of coupling term}

In the absence of a coupling term $(\lambda=0)$, hexatic and nematic degrees of freedom undergo separate KT transitions at temperatures [1,17,89-91]

$$
\begin{aligned}
& T_{2, \mathrm{KT}}^{(\lambda=0)}(\Delta) / J=0.89 \Delta, \\
& T_{6, \mathrm{KT}}^{(\lambda=0)}(\Delta) / J=0.89(2-\Delta) .
\end{aligned}
$$

The resulting phase diagram is shown in Fig. 3 and exhibits four phases. For details of the classical Monte Carlo simulations used to obtain this phase diagram, see Sec. IV. These phases distinguish regions with short-range order of the hexatic and nematic degrees of freedom from regions with QLRO. The phase transitions lie in the KT universality class and are thus characterized by a sudden jump of hexatic $(p=6)$ or nematic $(p=2)$ spin stiffness $K_{p}=\rho_{p} / T$ from zero to the universal value $K_{p}\left(T_{p, \mathrm{KT}}\right)=2 / \pi$. The background color in Fig. 3 shows that the specific heat $c$ exhibits a broad hump above the transition at about $T=1.1 T_{\mathrm{KT}}$ [17]. In the isotropic limit $\Delta=1$, the two KT transitions occur at the same temperature: $T_{\mathrm{KT}}^{(\lambda=0)}=0.89 \mathrm{~J}$.

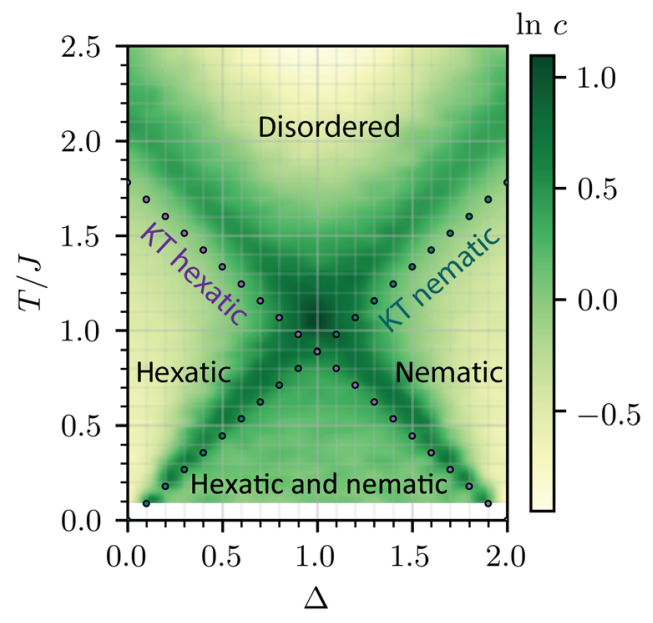

FIG. 3. Numerical phase diagram of the uncoupled $\lambda=0$ hexatic-nematic XY model in Eq. (3.1) as a function of the temperature $T$ and ratio of exchange couplings $\Delta=J_{2} / J$ obtained from classical Monte Carlo simulations. Background color density denotes specific heat per site $c$ at system size $L=$ 40 (see logarithmic color bar). We distinguish four different phases: (i) a disordered phase with purely short-range correlations, (ii) a hexatic phase with algebraic correlations of $\left\langle\cos \left(\theta_{i}-\theta_{j}\right)\right\rangle \sim|i-j|^{-\eta_{6}(T)}$, (iii) a nematic phase with algebraic correlations of $\left\langle\cos \left(\phi_{i}-\phi_{j}\right)\right\rangle \sim|i-j|^{-\eta_{2}(T)}$, and (iv) an ordered phase with algebraic correlations of both hexatic and nematic degrees of freedom. Here, $\langle\mathcal{O}\rangle$ denotes the thermal average. The KT transition temperatures (purple and blue dots) are obtained from the universal stiffness jump criterion $\rho^{(p)}\left(T_{p, \mathrm{KT}}\right)=$ $2 T_{p, \mathrm{KT}} / \pi$ for nematic $p=2$ and hexatic $p=6$ variable extrapolated to infinite system size.

In order to determine the effect of a finite hexaticnematic coupling term $(\lambda>0)$ on the phase diagram, we calculate its renormalization-group (RG) scaling dimension $\mathcal{D}_{\lambda}$. This can be done straightforwardly at $\Delta=1$ and $T_{\mathrm{KT}}$, where free vortex excitations are absent, to yield

$$
\mathcal{D}_{\lambda}=2-\frac{1}{4 \pi}\left(\frac{1}{K_{R, 6}}+\frac{9}{K_{R, 2}}\right)=\frac{3}{4} .
$$

Here, we use that $K_{R, p}=2 / \pi$ is the exact value of the renormalized spin stiffness at the KT transition temperature $T_{p, \mathrm{KT}}^{(\lambda=0)}$ [17]. Note that $K_{p}$ is independent of $p$ only for the rescaled Hamiltonian in Eq. (3.1), where the minimal phase winding of both hexatic and nematic vortices is equal to $2 \pi$, but the final result $\left(\mathcal{D}_{\lambda}=3 / 4\right)$ is identical if one uses Eq. (2.1). Further details of this derivation are presented in the Appendix A.

A positive scaling dimension $\mathcal{D}_{\lambda}>0$ indicates that $\lambda$ is a relevant perturbation at $T_{\mathrm{KT}}^{(\lambda=0)}$ and will drive the system away from the uncoupled KT fixed point. This suggests that the system develops long-range Potts order above the KT transition, $T_{\mathbb{Z}_{3}}>T_{\mathrm{KT}}^{(\lambda=0)}$. Certainly, as $\lambda$ grows toward 
longer length scales, hexatic and nematic angles are forced to arrange into one of the three parallel configurations shown in Fig. 2(d) for the equivalent $\vartheta$ and $\varphi$ variables. The constraint due to $\lambda$, as expressed for Eq. (3.1), is then

$$
\theta_{i}-3 \phi_{i}=2 \pi n_{i} \Rightarrow \phi_{i}=\frac{\theta_{i}}{3}+\frac{2 \pi n_{i}}{3} .
$$

Here, $n_{i}=-1,0,1$ is a $\mathbb{Z}_{3}$ degree of freedom. A "centerof-mass" variable $\kappa_{i}$ corresponding to the global $\mathrm{O}(2)$ rotations can also be defined as

$$
\frac{\theta_{i}+3 \phi_{i}}{2}=\kappa_{i}
$$

Note that if the locking constraint is fulfilled, the model (3.1) becomes equivalent to a generalized XY model [14,15], in our case for $q=3$ [92-94]. We review the results of the fully locked model in Sec. III D. The locking transition occurs as a crossover at a temperature $T_{\lambda}(\lambda)$ that depends on $\lambda$. Below this temperature, the nematic and hexatic fields are isotropic, but they are locked with one another, and the system is effectively described by the $n_{i}$ and $\kappa_{i}$ center-of-mass fields. Since $\mathcal{D}_{\lambda}>0$, it follows that $T_{\lambda}>T_{\mathrm{KT}}$ for all nonzero values of $\lambda$. For small initial values of $\lambda \ll 1, T_{\lambda}$ can be estimated from analyzing the $\mathrm{RG}$ equation of an $\mathrm{XY}$ model in a threefold potential, which yields $T_{\lambda} \approx[(8 \pi) / 9] J$ [76]. For large initial values of $\lambda \gg 1$, the locking occurs at a temperature $T_{\lambda} \gg J$. For $T<T_{\lambda}$, the symmetry of the model is lowered from $\mathrm{O}(2) \times \mathrm{O}(2) \rightarrow \mathrm{O}(2) \times \mathbb{Z}_{3}$. Note that if one adopts this formalism to other competing periodicities, one generically will find $\mathcal{D}_{\lambda}>0$, so that for $T<T_{p, \lambda}$, the symmetry of the model is lowered from $\mathrm{O}(2) \times \mathrm{O}(2) \rightarrow \mathrm{O}(2) \times \mathbb{Z}_{p}$. Although this seems like a general principle, it was shown that the situation with $p \geq 4$ does not present a classical Potts $\mathbb{Z}_{p}$ phase transition [76], and we do not expect the same arguments presented here to hold there. As we are motivated by a specific experimental setup, a systematic study of systems with competing periodicity is beyond the scope of this work.

\section{B. Vortex excitations in the coupled model}

For $T<T_{\lambda}$, the relative angle between nematic and hexatic degrees of freedom is a $\mathbb{Z}_{3}$ variable $n_{i}$. Being discrete, $n_{i}$ can develop LR order at a finite transition temperature $T_{3}$. The central question is whether $T_{3}$ lies above or below the KT transition of the center-of-mass $\mathrm{O}$ (2) variable defined in Eq. (3.5). If $T_{3}>T_{\mathrm{KT}}$, the situation corresponds to the experimentally observed order of phase transitions in 54COOBC. This turns out to be a rather delicate issue $[34,35]$ that requires a careful and unbiased large-scale computational effort, which we describe in Secs. IV and V. We note that several studies of related coupled XY models obtained by taking the limit of $\lambda \rightarrow \infty$ in Eq. (3.1) revealed intriguing behavior close to $T_{\mathrm{KT}}$ at $\Delta=1$ [93-98]. In this section, we give analytical arguments that reveal the subtleties which arise when addressing this question.

Vortex excitations in the system are extremely important, as their binding (unbinding) is related to the KT ordered (disordered) phases. Let us now discuss their role, and in particular, the impact of nematic-hexatic phase locking at $T<T_{\lambda}$ as described by Eq. (3.4) on their formation. For simplicity, we focus on the isotropic point $\Delta=1$, but similar arguments can be given for other values of $\Delta$. It is advantageous to refer to Hamiltonian (2.1), which lends itself to a clean interpretation of the vortex defects. We provide a lexicon for the vortex excitations for the Hamiltonian of Eq. (3.1), which is used extensively for technical reasons in the following sections, in Table I. The same arguments can be made for the model of Eq. (2.4) in the original variables, for which the minimal winding around a nematic (hexatic) vortex is given by $\pi(2 \pi / 6)$, hence, their respective names. For simplicity, we use $\lambda$ whenever we refer to the hexatic-nematic coupling term, irrespective of the particular model.

In the absence of the coupling term $(\lambda=0)$, hexatic and nematic systems undergo independent KT transitions (Fig. 3). In the language of Hamiltonian (2.1), the minimal charge of a hexatic (nematic) vortex is $q_{\vartheta}=1 / 3\left(q_{\varphi}=1\right)$ corresponding to a phase winding of $2 \pi q_{\vartheta}$ and $2 \pi q_{\varphi}$ around a vortex, respectively. Thus, at the KT transition, point vortices of charge $q_{\varphi}= \pm 1\left(q_{\vartheta}= \pm 1 / 3\right)$ unbind in the nematic (hexatic) system. Free vortices limit the correlation lengths $\xi_{6}\left(\xi_{2}\right)$ for $\vartheta(\varphi)$ to a finite value for all $T>T_{\mathrm{KT}}$. Vortices are bound into pairs of total charge zero in the critical phase for $T<T_{p, \mathrm{KT}}$, where the correlation lengths are infinite.

The situation is notably different at nonzero $\lambda$ and temperatures $T<T_{\lambda}$ below the locking crossover. While the domain of the angles reads $\vartheta \in\{0,[(2 \pi) / 3]\}$ and $\varphi \in[0,2 \pi)$, the locking condition imposes a distinct constraint on the phase winding around nematic and hexatic vortices, such that

$$
q_{\vartheta} \equiv q_{\varphi}+\frac{\Delta n}{3} \quad(\bmod 1), \quad(\lambda \neq 0)
$$

where $\Delta n$ counts the changes in the Potts index $n$ as one loops around a vortex core. Note that the modulo operation originates from the definition of the Potts variables $n$, such that $n=-2 \equiv 1$.

If the system is Potts disordered, one expects a sample to be swarmed by a network of domain walls of the $\sigma=$ $[(2 \pi) / 3] n$ variable, as domains get smaller and smaller for temperatures above a Potts ordering temperature $T_{3}$. For such a system with global Potts disorder, $\Delta n \neq 0$ generically. Hence, the solution of Eq. (3.6) leads to $\frac{1}{3} \equiv 0+\frac{1}{3}$, i.e., domain walls where $\Delta n=1$ are necessarily attached to 
hexatic vortices. These domain walls are energetically costly due to the significant nematic gradient energy that arises across them. Proliferation of these vortices and their eventual unbinding leads to a hexatic-KT transition above the $T_{3}$ transition. This is the mechanism at play at small $J_{2} / J_{6}$ [or, alternatively, for small $\Delta$ in Eq. (3.1)], as can be seen in Fig. 1 on the left side.

In the presence of Potts order, one has that globally, $\Delta n=0$. The solution to Eq. (3.6) is then $1 \equiv 1$ or $3\left(\frac{1}{3}\right) \equiv 1$. Both cases correspond to nematic vortices, but there is a subtle difference between the two. In the first case, a hexatic vortex of charge 3 times its elementary charge is at the same site as a nematic vortex. This is a nematic point vortex.

The alternative is for the nematic vortex to correspond to a triad of hexatic vortices. Since each hexatic vortex is attached to a Potts domain wall, one simple way to solve such a triad is to link all three hexatic vortices via their domain wall, as can be seen in Fig. 1(e). In essence, the domain walls act as a binding force for the fractional vortices, i.e., the hexatic vortices, as they imply locally fractional nematic vortices. Such a split vortex can be much more extended than its point vortex counterpart. In Sec. III C, we provide an analytical argument that extended nematic vortices are lower energy than their point vortex. Even though the domain walls can be quite energetic, vortices of charge triple that of their elementary charge are extremely costly due to the phase winding around the vortex core.

Note that if one uses the convention of Eq. (3.1), which is numerically advantageous, where both $\theta$ and $\phi$ are within the interval $[0,2 \pi)$, the minimal phase winding around a vortex is given by $2 \pi$ in both cases, corresponding to charge $q_{\theta}=1$ and $q_{\phi}=1$ elementary vortices. The relative locking between hexatic and nematic angles is described then by Eq. (3.4), leading to the constraint that

$$
\frac{q_{\theta}}{3} \equiv q_{\phi}+\frac{\Delta n}{3} \quad(\bmod 1), \quad(\lambda \neq 0)
$$

Thus, a nematic vortex with minimal $2 \pi$ phase winding must be accompanied by a hexatic vortex of charge $q_{\theta}=3$ that exhibits a phase winding of $2 \pi q_{\theta}=6 \pi$. In both descriptions, a nematic vortex of minimal charge $q_{\phi}$ necessarily pairs with a hexatic vortex whose charge is 3 times larger than its minimal charge. The hexatic vortex is then a situation where $q_{\theta}=1$ and $\Delta n=1$, which means that there is a Potts domain wall due to the $2 \pi / 3$ mismatch in the $\phi$ variable. Similarly, the composite nematic vortex has $q_{\phi}=1$ and three $q_{\theta}=1$ vortices, such that $(1+1+1) / 3 \equiv 1$, with each hexatic vortex confined via the attractive domain wall. For all of the following sections, we use the model presented in Eq. (3.1). Translation between the different vortex formalisms [Eq. (2.1) vs Eq. (3.1)] can be done through Table I provided in this subsection.
TABLE I. Lexicon between the different notations of Eqs. (2.1) and (3.1), establishing the link between the two conventions. The elementary vortex charges are added in the first two lines. The expressions of the last line are understood modulo 1 .

\begin{tabular}{lcc}
\hline \hline Model & Eq. $(2.1)$ & Eq. $(3.1)$ \\
\hline Hexatic & $\vartheta(\bmod [(2 \pi) / 3])$ & $\theta(\bmod 2 \pi)$ \\
& $q_{\vartheta}= \pm 1 / 3$ & $q_{\theta}= \pm 1$ \\
Nematic & $\varphi(\bmod 2 \pi)$ & $\phi(\bmod 2 \pi)$ \\
& $q_{\varphi}= \pm 1$ & $q_{\phi}= \pm 1$ \\
$2 \pi n_{i} \equiv$ & $3\left(\vartheta_{i}-\varphi_{i}\right)$ & $\theta_{i}-3 \phi_{i}$ \\
Vortex locking & $q_{\vartheta} \equiv q_{\varphi}+[(\Delta n) / 3]$ & {$\left[\left(q_{\theta}\right) / 3\right] \equiv q_{\phi}+[(\Delta n) / 3]$} \\
\hline \hline
\end{tabular}

In other words, in this model, the transition to the relative Potts phase corresponds to the confinement transition of fractionalized $q_{\phi}=1 / 3$ nematic vortices, which drives the formation of a bound state of the three attached elementary $q_{\theta}=1$ hexatic vortices through the constraint of Eq. (3.7). In the absence of free elementary hexatic vortices, a Potts order parameter can be well defined even in the presence of nematic vortices, as the potential $\lambda$ leads to the hexatic variable acting as a triaxial field for the nematic degrees of freedom. In contrast, no independent $\mathbb{Z}_{3}$ degree of freedom exists below the nematic-KT transition, as the potential $\lambda$ leads to the nematic variable acting as a uniaxial field for the hexatic degrees of freedom. Furthermore, elementary hexatic vortices are attached to "dangling" domain walls which, if they were not confined, would fully destroy the LR Potts order that is set in the system. The composite vortices of total elementary nematic charge are then the only ones allowed in the relative $\mathbb{Z}_{3}$ ordered state. We cover the energetics of the extended nematic vortices in the following subsection.

This transition is expected to lie in the 2D Potts universality class, which is characterized by the critical exponents $\alpha=1 / 3, \beta=1 / 9, \gamma=13 / 9, \nu=5 / 6$ [99]. Note in particular that the specific heat exponent $\alpha$, such that $c \propto t^{-\alpha}$, leads to a pronounced divergence of the specific heat distinct from the smooth behavior observed for KT transitions with a broad hump at about $1.1 T_{\mathrm{KT}}$ [17]. We exploit this notable difference to distinguish between Potts and KT transitions in our Monte Carlo simulations in Sec. V.

\section{Vortex fractionalization and the extended vortices}

Nematic $q_{\phi}=1$ vortices, which are accompanied by a $q_{\theta}=3$ hexatic vortex, are the free "elementary excitations" in the system at temperatures below $T_{\lambda}$. Lower-charge vortices are held together by domain-wall strings with finite tension, arising from the gradient energy cost of the domain wall due to the "fractional" hexatic vortex they contain. 
This observation immediately questions the possibility that a KT transition can take place below the Potts transition, because pointlike vortices are expected to bind at a temperature above a possible Potts transition $T_{3}$. Specifically, setting $\nabla \vartheta=\nabla \varphi$ once below $T_{\lambda}$ [alternatively, setting $\nabla \theta=9 \nabla \phi$ due to Eq. (3.4)] leads to $T_{\mathrm{KT}, \mathrm{nem}}^{\lambda \neq 0}=10 T_{\mathrm{KT}}^{\lambda=0} \approx$ $[(10 \pi) / 2] J$. The estimated KT transition temperature is thus even larger than $T_{\lambda} \approx[(8 \pi) / 9] J$ (which bounds $T_{3}$ in the small- $\lambda$ regime).

This estimate, however, leaves out the possibility of vortex fractionalization and the emergence of extended vortices [56,57]. Indeed, it is well known that higher charge vortices can reduce their (gradient) energy by splitting into multiple lower-charge objects. In addition, hexatic and nematic degrees of freedom are continuous; therefore, the domain walls separating regions with different Potts variables $n_{i}$ can acquire a finite width $\xi_{\mathrm{DW}}$. The width $\xi_{\mathrm{DW}}$ is determined by a balance between the cost of violating the locking constraint in Eq. (3.4) imposed by the $\lambda$ term and the gain in gradient energy by distributing the angle mismatch over a finite length. The width of the domain walls approaches the minimal size of the lattice constant only as the renormalized $\lambda \gg 1$. For example, splitting a $q_{\theta}=3$ vortex into three $q_{\theta}=1$ vortices reduces the gradient energy by a factor of $3^{2}-3=6[6]$ [similarly, splitting a $q_{\vartheta}=1=3\left(\frac{1}{3}\right)$ vortex]. Previous studies of continuous models with the same $\mathrm{O}(2) \times \mathbb{Z}_{3}$ symmetry have shown that vortex splitting leads to a lowering of the system's energy [8,59,62-64].

Here, this splitting, however, implies fractionalization of the joint nematic vortex into three vortices of fractional charge $q_{\phi}=1 / 3$, held together by domain-wall strings where $\Delta n= \pm 1$. The competition of gradient and domainwall energy results in an extended vortex core [6]. By comparing the energy of a point vortex $[3=3(1)]$ to a split vortex $[1+1+1=3(1)$, which looks like a point vortex at distances larger than the core], we arrive at the conclusion that it is energetically favored to split the vortex. For a circular geometry as in Fig. 1(e), this leads to an optimal radius of $R \simeq 2.5 a$ for the value of the coupling $\lambda$ used in this work, or a split vortex with an area 6.25 times greater than its point vortex counterpart. Thorough derivation of this result is presented in Appendix $B$. Importantly, the unbinding of extended vortices is known to occur at a temperature lower than the unbinding of pointlike vortices [58]. This can be understood from the fact that the initial value of the vortex fugacity increases by a factor of $(R / a)^{2}$, where $a$ is the microscopic lattice scale. For $R \gg a$, the KT transition temperature scales as $T_{\mathrm{KT}} \sim$ $1 / \ln \left[(R / a)^{2}\right]$ and is thus significantly reduced in the case of large vortex core sizes. The crucial open question is whether it is reduced to a value below the Potts transition temperature. To address this question in an unbiased way, we perform large-scale classical Monte Carlo simulations, which we discuss in the next section.

\section{Previous numerical studies}

Before delving into our numerical results, let us provide an overview of other numerical studies of coupled XY models. The particular one presented in Eq. (3.1) was first studied in two dimensions using Monte Carlo techniques [34,35], yet with a focus on a different region of the phase diagram $(\Delta \ll 1)$ and at substantially smaller system sizes than presented here $(L \leq 40)$. The sequence of transitions $T_{\mathrm{KT} \text {,hex }}>T_{\mathbb{Z}_{3}}$ was observed in that regime, which we also observe. In the regime of interest where $\Delta \simeq 1$, they concluded in a single transition where both nematic KT and Potts occurred simultaneously. We use that pioneering study as a benchmark for our simulations, and the improvement in algorithm and computing power combined with modern analytical insight reveals that those two transitions split in our study with the Potts transition occurring above the KT transition.

A three-dimensional version of the model was studied as well [100], but we do not expect the same behavior there, as the continuous phase transition is not KT-like, and vortex excitations are not the only relevant excitation at high temperature. A related coupled $X Y$ model with $\mathrm{O}(2) \times \mathbb{Z}_{2}$ symmetry instead of our $\mathrm{O}(2) \times \mathbb{Z}_{3}$ symmetry associated with atom-molecular mixing was studied extensively in two dimensions for problems associated with atommolecular mixing [7,101] and competing hexatic and threefold order parameters in smectic liquid crystals [102]. The $\Delta \simeq 1$ regime was, however, not the primary focus of those studies. Their conclusions for that regime was that a single transition occurred through which both discrete and continuous degrees of freedom became ordered simultaneously.

The community has been more focused on the infinite coupled limit $(\lambda \rightarrow \infty)$. Such generalized XY models can be written as

$$
\mathcal{H}_{\infty}=-\sum_{\langle i, j\rangle}\left[\Delta \cos \left(\phi_{i j}\right)+(2-\Delta) \cos \left(p \phi_{i j}\right)\right],
$$

where $\phi_{i j}=\phi_{i}-\phi_{j}$, with $p=3$ corresponding to the $\lambda \rightarrow \infty$ limit of Eqs. (2.1) and (3.1), and $p=2$ is obtained through the same limit for the atomic-molecular mixing model. These two models were studied via renormalization-group studies [4,14-16,60,61], Monte Carlo techniques [93-96,103,104], matrix-product states [98], and bosonization $[97,105]$. They all share a common phase diagram structure [106], with a well-understood regime at $\Delta \ll 1$ with a $p$-state discrete transition at $T_{p}$ (Ising or Potts) below a Kosterlitz-Thouless transition at $T_{\mathrm{KT}}$ where fractional defects unbind. For $\Delta \simeq 1$, the available evidence supports a single transition temperature corresponding to both a confinement transition of fractional vortices and a binding of integer-charge vortices [60,61,97]. From our analysis of the composite vortex in Sec. III C, we find that 
there is no gain at $\lambda \rightarrow \infty$ for an extended vortex over a point one. This would likely collapse the two transitions we find at finite $\lambda$ into one in that limit, such that they would be inseparable with current numerical accuracy. This is partly why our numerical study is focused on the coupled model in the intermediate regime, so as to see the postulated effect of the formation of composite vortices.

We note that in the case of the fully frustrated XY model (FFXY), which represents a periodic array of Josephson junctions with half a quantum flux through it, one rather has two $\mathrm{O}(2)$ order parameters coupled via a $\cos \left[2\left(\theta_{i}-\phi_{i}\right)\right]$ term. For this model, extensive analytical [4,5,107-109] and Monte Carlo simulations [89,110-116] were able to distinguish a clear regime where the relative $\mathbb{Z}_{2}$ symmetry breaking happens at a temperature higher than the global KT transition. It was also shown that the presence of the Ising line above the KT transition leads to the prospect of an emergent supersymmetry at the Ising-KT multicritical point [117]. This success was due to the power of modern implementations of Monte Carlo algorithms, as well as the increasing computing power available, further motivating

(a)

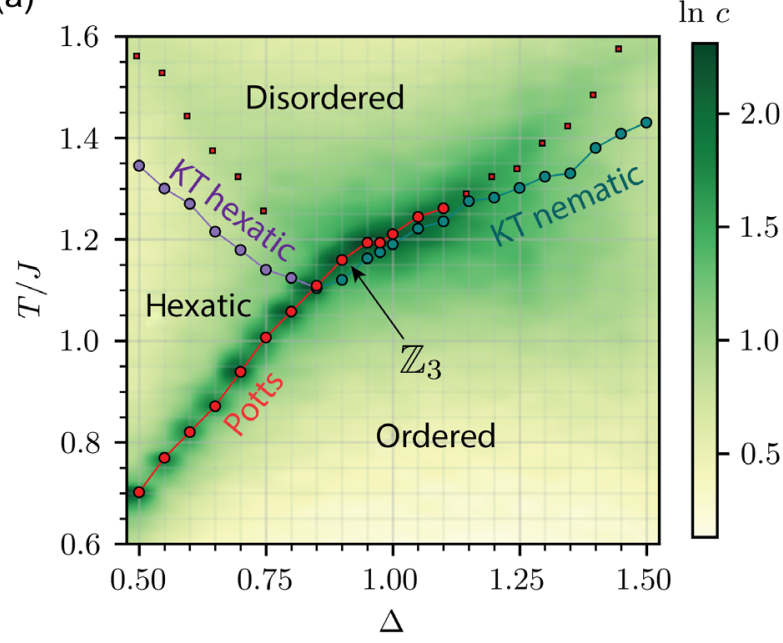

our reexamination of this classic hexatic-nematic problem of Eq. (3.1).

\section{MONTE CARLO SIMULATION: ALGORITHM AND OBSERVABLES}

In this section, we describe the algorithm used for our large-scale parallel-tempering Monte Carlo (MC) simulations of the model in Eq. (3.1). We give details in Sec. IV A and introduce the various observables that are measured to obtain the finite $\lambda$ phase diagram presented in Fig. 4 in Sec. IV B. The detailed investigation of the full phase diagram is presented in Sec. V. All data and code needed to generate the results in this paper are available online [118].

\section{A. Technical details of the Monte Carlo algorithm}

The employed MC algorithm combines a standard parallel-tempering update $[119,120]$ with a generalized Wolff cluster algorithm adapted to coupled XY models $[7,121]$. The simulations are performed on a square lattice with periodic boundary conditions of $N=L \times L$ sites,

(b)

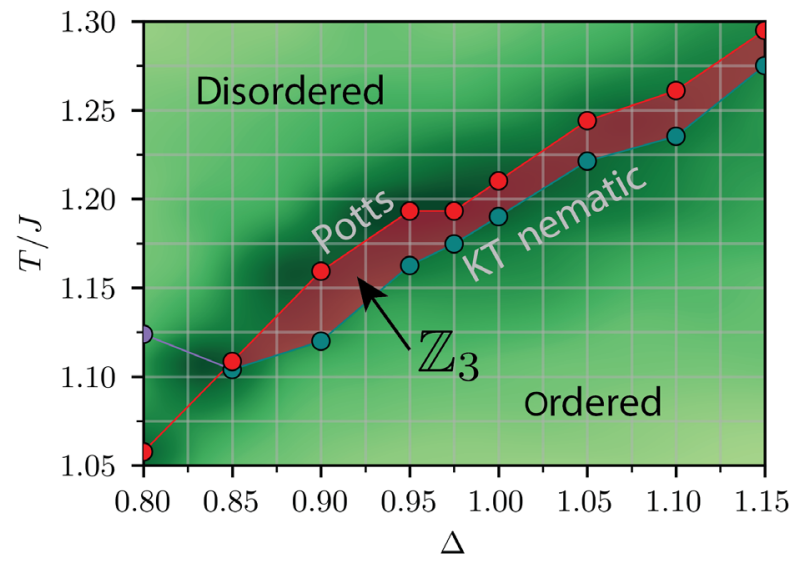

FIG. 4. (a) Numerical phase diagram of the coupled hexatic-nematic XY model in Eq. (3.1) as a function of the temperature $T$ and ratio of exchange couplings $\Delta=J_{2} / J$ obtained from classical Monte Carlo simulations. The hexatic-nematic coupling strength is set to $\lambda=2.1$ and $J=\frac{1}{2}\left(J_{2}+J_{6}\right)$. Background color density denotes specific heat per site $c$ at system size $L=40$ (see logarithmic color bar). We distinguish four different phases: (i) a disordered phase with purely short-range correlations, (ii) a hexatic phase with algebraic correlations of $\left\langle\cos \left(\theta_{i}-\theta_{j}\right)\right\rangle \sim|i-j|^{-\eta_{6}}$, (iii) a $\mathbb{Z}_{3}$ Potts phase with long-range correlations $\left\langle\cos \left(\sigma_{i}-\sigma_{j}\right)\right\rangle \rightarrow$ constant $>0$ as $|i-j| \rightarrow$ $\infty$ with $\sigma_{i}=\left(\theta_{i}-3 \phi_{i}\right) / 3$ but short-range correlations of hexatic and nematic angles, and (iv) an ordered phase with algebraic correlations of both hexatic and nematic degrees of freedom and long-range Potts correlations. Here, $\langle\mathcal{O}\rangle$ denotes the thermal average. The KT transition temperatures (blue and purple dots) are obtained from the total stiffness jump criterion. The size of the jump is used to distinguish between a nematic-KT transition, where $\rho\left(T_{\mathrm{KT}}\right)=2 T_{\mathrm{KT}} / \pi$ (unbinding of $q_{\phi}=1$ vortices, blue), and a hexatic-KT transition, where $\rho\left(T_{\mathrm{KT}}\right)=18 T_{\mathrm{KT}} / \pi$ (unbinding of $q_{\theta}=1$ vortices, purple). Note that we present the extrapolated KT critical temperatures to infinite system size (Fig. 6). The Potts transition temperature $T_{3}$ (red dots) is determined from the maximum of the specific heat $c$ at $L=40$, except at $\Delta=0.95,1.0$, where $T_{3}$ is obtained by fitting $c$ to the Potts scaling form $c=t^{-\alpha}$ with $t=$ $\left|T-T_{3}\right| / T_{3}$ and $\alpha=1 / 3$ (Figs. 5 and 6). Consistent Potts transition temperatures are obtained from a universal scaling analysis of the magnetization, susceptibility, and specific heat, as shown in Fig. 7. The red squares for $\Delta \geq 1.15$ track a broad maximum of $c$ associated with the KT nematic transition at $T_{2}$, demonstrating that the nature of the upper phase transition changes from Potts to KT at $\Delta=1.15$. On the left side of the phase diagram, the red squares correspond to the second, lower maxima of the specific heat corresponding the proliferation of hexatic vortices above the hexatic-KT transition. (b) Enlargement of the region with relative $\mathbb{Z}_{3}$ Potts order. The Potts phase exists for $0.9 \leq \Delta \leq 1.15$ and extends over a region $\Delta T / J \approx 0.005$. 
with $L$ ranging from 8 to 380 . For system sizes $L \leq 200$ ( $L>200$ ), we simulate 40 (64) configurations in parallel at different, geometrically spaced temperatures between $T_{\min }=0.5 \mathrm{~J}$ and $T_{\max }=1.6 \mathrm{~J}$ to obtain a general overview of the phase diagram. When looking directly at the nature of a phase transition at $T_{3}$, we always use a new temperature range such that $\left(T_{\min }, T_{\max }\right)=\left(0.95 T_{3}, 1.05 T_{3}\right)$, therefore increasing the resolution in temperature. The generalized Wolff algorithm takes into account that due to the coupling term $\lambda \sum_{i} \cos \left(\theta_{i}-3 \phi_{i}\right)$, one must flip the hexatic and nematic angles $\theta_{i}$ and $\phi_{i}$ in an anisotropic manner in order to explore both global $\mathrm{O}(2)$ and $\mathbb{Z}_{3}$ symmetries. The Wolff clusters are therefore built as follows: One first starts by randomly selecting a flip direction $\eta \in[0,6 \pi)$, a site $i$, and the type of variable $\theta_{i}$ or $\phi_{i}$. Depending on which variable is chosen, we then apply the flip rule

$$
\begin{aligned}
\theta_{i} \rightarrow \theta_{i}^{\prime} & =-\theta_{i}+\eta, \\
\phi_{i} \rightarrow \phi_{i}^{\prime} & =-\phi_{i}+\frac{\eta}{3} .
\end{aligned}
$$

Subsequently, one grows the cluster by investigating all five bonds connected to the chosen variable at site $i$. These connect to the four nearest neighbors of the same variable and to the other variable at the same site $i$. For each of these bonds, one calculates the energy cost $\Delta E$ of flipping the other member of the bond as well. This member is flipped, and the site is added to the cluster with probability $p=1-\min \{1, \exp (-\beta \Delta E)\}$, where $\beta=1 / T$. One then proceeds in the same way for all new members of the cluster until all outgoing bonds of the cluster are considered. Note that the clusters invade both $\left\{\theta_{i}\right\}$ and $\left\{\phi_{i}\right\}$ variables. At high temperatures, clusters barely spread through the system, whereas as $T \rightarrow 0$, the cluster size is of the order of $N$.

We perform the simulations for at least $4 \times 10^{5} \mathrm{MC}$ steps, where each step consists of a parallel-tempering update and a series of generalized Wolff moves, such that approximately $N$ sites are updated. To ensure thermalization, we discard the first half of the obtained configurations and measure the thermodynamic observables only during the second half of the MC steps. We introduce the different observables that we measure next. Finally, error bars are obtained using the standard jackknife method [122].

\section{B. Observables, phases, and phase transitions}

To distinguish the different phases of the model, we investigate several thermodynamic observables that can be grouped into three classes. First, we measure observables related to the energy fluctuations in the system, the specific heat per site $c$, and the Binder cumulant of the energy $B_{E}$ :

$$
\begin{aligned}
c & =\frac{\left\langle\mathcal{H}^{2}\right\rangle-\langle\mathcal{H}\rangle^{2}}{N T^{2} J^{2}}, \\
B_{E} & =\frac{\left\langle\mathcal{H}^{4}\right\rangle}{\left\langle\mathcal{H}^{2}\right\rangle^{2}}-1,
\end{aligned}
$$

where $\mathcal{H} / J$ is defined in Eq. (3.1). A characteristic signature of second-order phase transitions is strong fluctuations of the energy close to the critical point. The specific heat diverges as $c \propto t^{-\alpha}$ with $t=\left|T-T_{c}\right| / T_{c}$, and $B_{E}$ exhibits a sharp local maximum at $T_{c}$ [123-126]. In contrast, energy fluctuations are much less pronounced and more broadly distributed at a KT transition. Specifically, at a temperature of about $T=1.1 T_{\mathrm{KT}}$, the specific heat shows a rounded bump and $B_{E}$ exhibits a change in slope, which signals the thermal generation of vortex excitations. Both observables $c$ and $B_{E}$ are, therefore, well suited to distinguish between the second-order Potts phase transition (of $n_{i}$ ) and the KT transitions of hexatic and nematic variables $\theta_{i}$ and $\phi_{i}$. In particular, the sustained presence of a sharp local maximum in $B_{E}$ for all system sizes is a clear signature of a second-order phase transition.

The second class of observables we study are magnetizations and their susceptibilities that characterize the different phases. The three measured quantities are

$$
\begin{aligned}
& M_{\theta}=\frac{1}{N} \sum_{i} \cos \theta_{i}, \\
& M_{\phi}=\frac{1}{N} \sum_{i} \cos \phi_{i}, \\
& M_{\sigma}=\frac{1}{N} \sum_{i} \cos \sigma_{i} .
\end{aligned}
$$

Here, we introduce the $\mathbb{Z}_{3}$ Potts variable

$$
\sigma_{i}=\frac{2 \pi}{3} n_{i}=\frac{1}{3}\left(\theta_{i}-3 \phi_{i}\right),
$$

such that the hexatic-nematic coupling Hamiltonian term reads $\lambda \cos \left[3 \sigma_{i}\right]$. We also measure the associated magnetizations, susceptibilities, and Binder cumulants $(a=\theta, \phi, \sigma)$ :

$$
\begin{aligned}
m_{a} & =\left\langle M_{a}\right\rangle, \\
\chi_{a} & =\frac{N}{T}\left(\left\langle M_{a}\right\rangle^{2}-\left\langle M_{a}^{2}\right\rangle\right), \\
B_{a} & =1-\frac{\left\langle M_{a}^{4}\right\rangle}{3\left\langle M_{a}^{2}\right\rangle^{2}} .
\end{aligned}
$$

Below we perform a scaling analysis of $m_{a}, \chi_{a}$, and $c$ in order to extract the critical exponents of the observed second-order phase transition. The Binder cumulants of the magnetizations 
undergo steplike transitions from a value of $1 / 3$ at high temperature to a value of $2 / 3$ at low temperature at both KT and second-order phase transitions.

Finally, the third class of observables that we investigate is spin stiffnesses $\rho_{\alpha}$, which describe the free-energy change of the system under a uniform phase twist (along a given lattice direction $\alpha$ ). When hexatic and nematic variables are coupled to each other by nonzero $\lambda$, one cannot separate contributions arising from each individual variable and thus can extract only the total stiffness of the system. In order to fulfill the potential term constraint (3.4), we apply a uniform phase twist of the form

$$
\left(\phi_{i+\hat{\alpha}, i}, \theta_{i+\hat{\alpha}, i}\right) \rightarrow\left(\phi_{i+\hat{\alpha}, i}+\psi, \theta_{i+\hat{\alpha}, i}+3 \psi\right),
$$

where $\phi_{j i}=\phi_{j}-\phi_{i}, \theta_{j i}=\theta_{j}-\theta_{i}$, Here, $\psi$ is uniform across the system, and $\hat{\alpha}$ corresponds to either the $\hat{x}$ or $\hat{y}$ lattice direction. Note that the phase twist that is applied to the hexatic angle $\theta_{i}$ across each bond is 3 times larger than the twist applied to the nematic angle.

The spin stiffness at $T>0$ for an infinitesimal twist along direction $\alpha$ is defined as $\rho^{(\alpha)}=(1 / N)\left[\left(\partial^{2} F\right) /\left(\partial \psi^{2}\right)\right]$, and following a standard derivation $[127,128]$, one arrives at the explicit expressions

$$
\begin{aligned}
\rho & =\frac{1}{2}\left(\rho^{(x)}+\rho^{(y)}\right), \\
\rho^{(\alpha)} & =\frac{1}{N}\left\langle\mathcal{H}^{(\alpha)}\right\rangle-\frac{\beta}{N}\left[\left\langle\left(I^{(\alpha)}\right)^{2}\right\rangle-\left\langle I^{(\alpha)}\right\rangle^{2}\right], \\
\mathcal{H}^{(\alpha)} & =\Delta \sum_{\langle i, j\rangle_{\alpha}} \cos \phi_{i j}+9(2-\Delta) \sum_{\langle i, j\rangle_{\alpha}} \cos \theta_{i j}, \\
I^{(\alpha)} & =\Delta \sum_{\langle i, j\rangle_{\alpha}} \sin \phi_{i j}+3(2-\Delta) \sum_{\langle i, j\rangle_{\alpha}} \sin \theta_{i j} .
\end{aligned}
$$

Here we define the total stiffness $\rho$ that is averaged over both lattice directions. The summation $\langle i, j\rangle_{\alpha}$ runs over all bonds along direction $\alpha$. For $\lambda=0$, cross-correlations between hexatic and nematic variables are absent, and the total stiffness decomposes into the sum $\rho(\lambda=0)=$ $\rho_{2} \Delta+9 \rho_{6}(2-\Delta)$, where $\rho_{2}\left(\rho_{6}\right)$ is the stiffness of the uncoupled nematic (hexatic) system. Note that the factor of 9 in the $\rho_{6}$ part is due to the fact that while a uniform infinitesimal twist $\psi$ is applied on the nematic, the hexatic exhibits an infinitesimal twist of $3 \psi$.

It is well known that the discontinuous jump of the spin stiffness at the KT transition can be directly associated with the charge $q$ of the unbinding vortices (phase winding of $2 \pi q$ around the vortex) $[94,129,130]$. In particular, one finds

$$
\rho\left[T_{\mathrm{KT}, q}^{-}\right]=\frac{2 T_{\mathrm{KT}, q}^{-}}{\pi q^{2}}
$$

just below the transition, while the stiffness vanishes above the transition. The hexatic-nematic model of Eq. (3.1) supports two types of vortex excitations associated with the hexatic and nematic angles. Because of the choice we make in Eq. (4.6) to apply the phase twist uniformly on the nematic $\phi$ variables, the type of vortex unbinding in $\phi$ dictates the value of the stiffness jump. A KT transition dominated by the unbinding of $q_{\phi}= \pm 1$ vortices results in a normal jump of the total stiffness $\rho$ by $2 T_{\mathrm{KT}, 2} / \pi$. We thus denote a KT transition characterized by this jump value as "nematic KT" $T_{\mathrm{KT}, 2} \equiv T_{2}$. On the other hand, a KT transition driven by the unbinding of hexatic vortices of integer charge $q_{\theta}= \pm 1$ results in a jump of the total stiffness by $18 T_{\mathrm{KT}, 6} / \pi$. A KT transition characterized by this larger jump value is denoted as "hexatic KT" $T_{\mathrm{KT}, 6} \equiv T_{6}$. To summarize, the location of stiffness jumps is used to determine the KT transition temperature $T_{\mathrm{KT}}$, and the height of the jump provides clear evidence of the type of vortex unbinding that occurs across the transition.

In the following, we use these observables to identify the different phases and phase transition universality classes presented in Fig. 4.

\section{COMPUTATIONAL RESULTS}

Here we present the results of extensive MC simulations of the model (3.1). Our main result is the phase diagram shown in Fig. 4 as a function of the temperature $T$ and coupling constant ratio $\Delta=J_{2} / J$ with $J=\frac{1}{2}\left(J_{2}+J_{6}\right)$. The phase diagram is obtained for fixed hexatic-nematic coupling strength $\lambda=2.1$. Crucially, it shows a region near to $\Delta=1$, where the Potts phase transition $T_{3}$ lies above the nematic-KT transition $T_{\mathrm{KT} \text {,nem }}$, which is highlighted in Fig. 4(b). Although the two transitions occur close to each other and the ratio of the transition temperatures reads $T_{3} / T_{\mathrm{KT}}(\Delta=1)=1.007$, the detailed numerical analysis presented below shows that $T_{3}>T_{\mathrm{KT}}$ with a high degree of statistical confidence. This numerically demonstrates that LR order in the emergent Potts $\mathbb{Z}_{3}$ variable $n_{i}$, as defined related to $\sigma_{i}$ in Eq. (4.4), exists even in a region with finiterange correlations of the hexatic and nematic degrees of freedom. This suggests that the hidden order phase that is experimentally observed in 54COOBC films is characterized by LR relative Potts order, and the sharp specific heat divergence is associated with a 2D Potts phase transition. In the following subsections, we separately discuss the different regions in the phase diagram in the order of increasing values of $\Delta$.

\section{A. Upper KT and lower Potts transition at $\boldsymbol{\Delta}<0.9$}

As shown in the phase diagram in Fig. 4(a), for $\Delta<0.9$ and starting from the disordered high-temperature phase, the system first develops hexatic QLRO across a KT transition at temperature $T_{6}$. This transition corresponds 

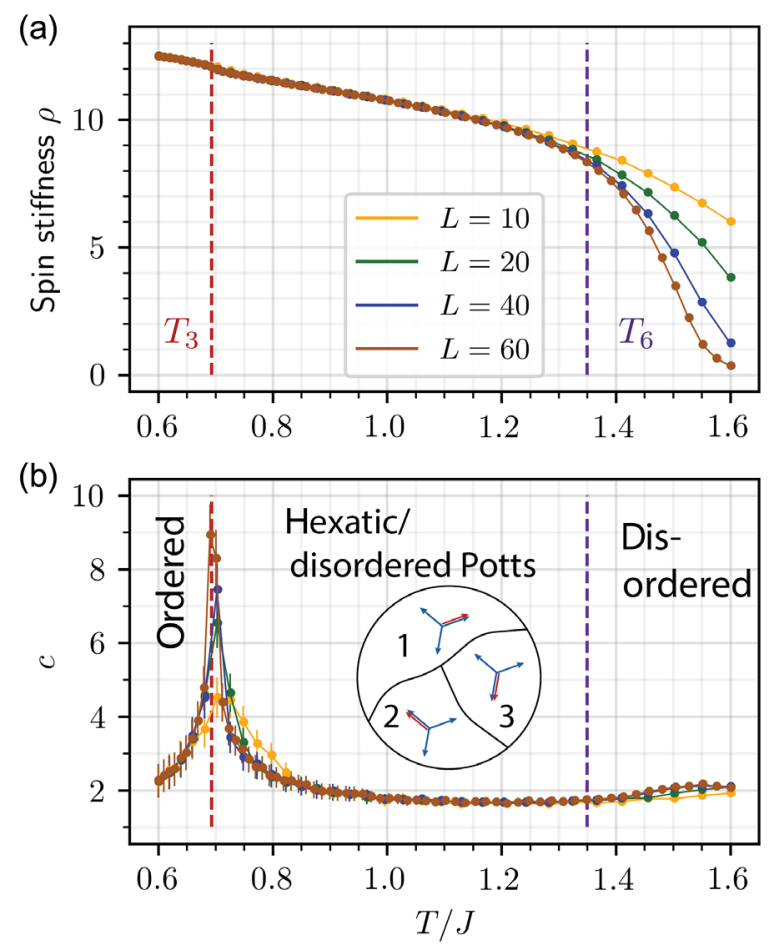

FIG. 5. Thermodynamic observables at $\Delta=0.5$ for systems with linear sizes up to $L=60$, replicating the results of Ref. [34] which is used as a benchmark. (a) Total spin stiffness $\rho$ defined in Eq. (4.7) as a function of the temperature $T / J$. Upturn at $T_{6}$ signals KT transition into the hexatic phase. The transition temperature is determined most accurately using the universal jump criterion $\rho\left(T_{6}^{-}\right)=18 T_{6} / \pi$, which yields $T_{6}(\Delta=0.5)=1.35 \pm 0.01$. (b) Specific heat per site $c$ as a function of the temperature $T / J$. While $c$ exhibits a broad maximum above the hexatic-KT transition at $1.1 T_{6}$, it develops a sharp peak at the lower transition at $T_{3}$, which increases with system size $L$. This is characteristic of the Potts phase transition in 2D, where $c \sim t^{-\alpha}$ with exponent $\alpha=1 / 3$. We extract the transition temperature $T_{3}(\Delta=0.5)=0.693 \pm 0.005$ from the location of the maximum of $c$ for $L=60$. The inset depicts the three $\mathbb{Z}_{3}$ domains with different relative hexatic-nematic ordering in the hexatic phase. Long-range $\mathbb{Z}_{3}$ order develops only at the lower $T_{3}$ transition.

to a binding of hexatic vortices with charge $q_{\theta}= \pm 1$. Since $T_{6}<T_{\lambda}$, i.e., the temperature at which the $\lambda$ coupling becomes relevant, each hexatic vortex is found at the end of a domain-wall string and can also be thought of as a fractional nematic vortex of $q_{\phi}=1 / 3$, as shown in Fig. 1. As $T_{6}>T_{3}$, Potts domain walls have proliferated already at lower temperatures. As shown in Fig. 5(a), the binding of fractional nematic vortices results in a jump of the total stiffness equal to $\rho\left(T_{6}^{-}\right)=(18 / \pi) T_{6}$. We determine $T_{6}$ by extracting $T_{6}(L)$ for different system sizes $L$ using this criterion and then extrapolating to infinite system size. As shown in Fig. 5(b), the specific heat $c$ exhibits only a broad maximum at about $1.1 T_{6}$, as expected from a KT transition. In contrast, $c$ develops a pronounced peak at lower temperature $T_{3}$, which grows with system size $L$.
Such a power-law singularity is expected, for example, at a 2D Potts phase transition, where $c \sim t^{-\alpha}$ with $\alpha=1 / 3$ and $t=\left(T-T_{3}\right) / T_{3}$.

The inset of Fig. 5(b) schematically depicts the ordering that occurs at the lower transition (see also Fig. 1): In the hexatic phase, there exist different domains of the threestate Potts order parameter $n_{i} \in \mathbb{Z}_{3}$ [see Eq. (3.4)]. Regions with different $n_{i}$ are separated by domain walls that also represent domain-wall strings for the nematic angle, where $\phi$ winds by $2 \pi / 3$. There exist fractional vortices with $q_{\phi}=$ $1 / 3$ at the end of these strings. In this regime, the cost of these domain walls is set by the nematic gradient energy, which is proportional to $J_{2}$, which explains why $T_{3} \rightarrow 0$ as $\Delta \rightarrow 0$. The transition at $T_{3}$ corresponds to the ordering of the $\mathbb{Z}_{3}$ variable $n_{i}$, which occurs via a $2 \mathrm{D}$ Potts phase transition. Since $n_{i}$ is a discrete degree of freedom, the system exhibits true LR Potts order below $T_{3}$. This scenario of an upper hexatic-KT transition, where $\theta$ variables develop QLRO, and a lower Potts transition, at which $n_{i}$ develops LRO and $\phi$ QLRO, can also be observed in the Binder cumulants of the respective magnetizations, as shown in the left column of Fig. 10 in Appendix C. It is also clearly seen in the Binder cumulant of the energy $B_{E}$ shown in Fig. 8. At the lower transition at $T_{3}$, the Binder cumulant $B_{E}$ develops a sharp peak, which is a clear indication of a second-order phase transition. In contrast, close to the upper transition $T_{6}, B_{E}$ features only a change in slope at about $1.1 T_{6}$, which is known to correspond to its behavior close to a KT transition. The Binder cumulant $B_{E}$ is, therefore, a convenient way to distinguish a secondorder phase transition from a KT transition, as we discuss more below. Finally, we note that such an order of phase transitions (upper KT, lower Potts) has previously been reported for the generalized XY model [7,34,94] that corresponds to the $\lambda \rightarrow \infty$ limit of Eq. (3.1), as we mention in Sec. III D.

\section{B. Upper Potts and lower KT transition at $0.9 \leq \Delta<1.15$}

Let us now discuss the region of main interest in the phase diagram around $\Delta=1.0$. As illustrated by the Binder cumulants $B_{\theta}, B_{\phi}, B_{\sigma}$ in the middle column of Fig. 10 in Appendix $C$, the ordering of hexatic, nematic, and Potts degrees of freedom all occur at nearby temperatures in this region. As shown in Fig. 4, we find that the Potts transition temperature increases monotonically with increasing $\Delta$ while the KT transition decreases, until the three Binder cumulants cross at a value of $\Delta$ between 0.85 and 0.9 . For $0.9 \leq \Delta<1.15$, the Potts transition occurs above the KT transition. As the KT transition temperature exhibits a minimum at $\Delta=0.9$, both transitions track each other with similar slope in that region. While the separation of the two transitions is small $\left(T_{3}-T_{\mathrm{KT}}\right) / T_{3} \approx 1 \%$, we can resolve them within error bars using extensive Monte Carlo simulations up to linear system sizes of $L=380$. This is 
demonstrated in Fig. 6, which shows results for $\Delta=1.0$. Figure 6(a) shows the spin stiffness $\rho$ and the specific heat $c$ that are used to extract KT and Potts transition temperatures, respectively. Specifically, we extract $T_{3}(L)$ from a fit of $c(T, L) \propto t^{-\alpha}$ with $t=\left[T-T_{3}(L) / T_{3}(L)\right]$ and Potts exponent $\alpha=1 / 3$. As shown in detail in Fig. 6(b), we determine the KT transition temperature $T_{2}$ from the universal jump criterion $\rho(T, L)=2 T_{2}(L) / \pi$. We note that the nature of the KT transition changes from hexatic to nematic at $\Delta=0.9$. Figure 6(c) displays the resulting system-size-dependent transition temperatures $T_{2}(L)$ and $T_{3}(L)$. It can be clearly seen there that for system sizes $L>60$, the pseudo-critical temperatures $T_{2}(L)$ and $T_{3}(L)$ are consistently ordered such that $T_{2}(L)<T_{3}(L)$. We determine the transition temperatures in the thermodynamic limit by extrapolating to infinite system sizes using the expected scaling forms $T_{2}(L)-T_{2}(\infty)=a /(\ln L)^{2}$ and $T_{3}(L)-T_{3}(\infty)=a^{\prime} L^{-1 / \nu}$ with Potts correlation length exponent $\nu=5 / 6$. We find that $T_{3}(\infty)>T_{2}(\infty)$ within a confidence of more than 1 standard deviation, specifically $T_{3}(\infty)=1.2022 \pm 0.0005$ and $T_{2}(\infty)=1.194 \pm 0.002$ at $\Delta=1.0$. This is one of the main results of this work. In the thin region $T_{3}>T>T_{2}$, the system exhibits LR order in the relative hexatic-nematic orientation $n_{i}$ even though both angles are still only short-range correlated with a finite correlation length. We believe that this scenario of inverted Potts and KT transitions can explain the experimental observations in thin films of $54 \mathrm{COOBC}$, as we discuss above.
Once the system enters a region with LR Potts order, the only available asymptotically free vortex excitations are nematic $q_{\phi}=1$ vortices formed of three bound elementary $q_{\theta}=1$ vortices confined by the Potts domain wall's finite tension. A schematic representation of these objects is shown in Fig. 1(d). Unfortunately, a snapshot of the composite vortex within our MC simulations is not possible, as the fractional vortices will not necessarily confine with their nearest neighbor, creating a complex tangled web of hexatic fractional vortices. As per our analysis in Appendix B, the extent of composite vortices can be many lattice spacings. In the relative Potts phase, where a visual snapshot would be most beneficial, the high density of vortices leads to large overlap between the extended objects. As we discuss in Sec. III C, the energy cost of these nematic vortices can be significantly lowered at finite values of $\lambda$ by expanding the vortex core to host three hexatic vortices of $q_{\theta}=1$ [see Fig. 1(d)]. This decreases the transition temperature for the unbinding of such extended combined vortices by a factor of $1 / \ln [R / a]^{2}$, where $R>a$ is the vortex core size and $a$ is the lattice scale. As a result, the KT transition temperature for unbinding of nematic vortices may be pushed below $T_{3}$, as we observe in our MC simulations. We emphasize that while we focus on the value of $\lambda=2.1$ in our work, we indeed find a region with $T_{3}>T_{2}$ also for other values of $\lambda=0.5,1.0,4.0$, demonstrating that our conclusions hold for an extended regime of hexatic-nematic couplings. We suggest that
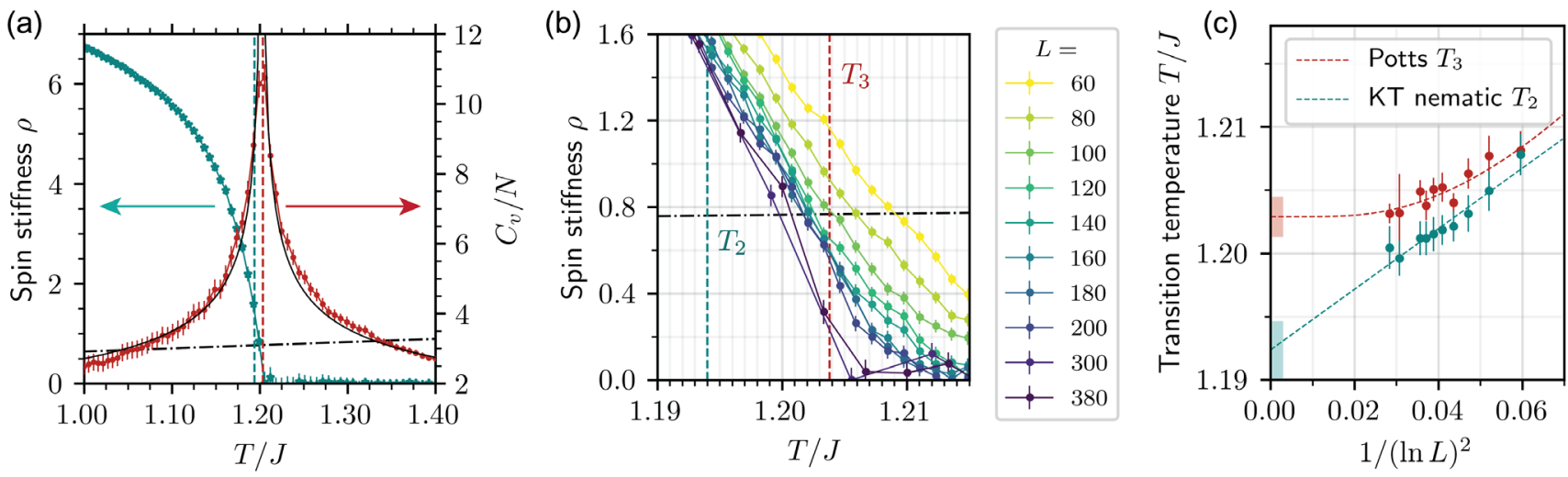

FIG. 6. (a) Spin stiffness $\rho$ and specific heat per site $c$ as a function of the temperature $T / J$ at $\Delta=1.0$ and for linear system size $L=300$. Dashed vertical lines denote the asymptotic transition temperature $T_{2} \equiv T_{2}(\infty)$ (blue) and $T_{3} \equiv T_{3}(\infty)$ (red) from extrapolation to $L \rightarrow \infty$ [see panel (c)]. The divergence of $c$ at $T_{3}$ occurs close to the upturn of $\rho$ at $T_{2}$. The solid black line is a fit of $c$ to $t^{-\alpha}$ with $\alpha=1 / 3$, which allows the extraction of a Potts transition temperature $T_{3}(L)$. The crossing of the dot-dashed black line $2 T / \pi$ with $\rho$ allows the KT transition temperature $T_{2}(L)$ to be determined. (b) Spin stiffness as a function of the temperature $T / J$ for $\Delta=1.0$ and different system sizes between $L=60$ and $L=380$. The crossing of $\rho$ with the dot-dashed black line at $T_{2}(L)$ shifts to lower temperatures as the system size $L$ increases. Dashed vertical lines follow the same convention as in panel (a). (c) Evolution of Potts $T_{3}(L)$ and nematic-KT $T_{2}(L)$ transition temperatures with system size $L$. Extrapolation to infinite system size is obtained by best fits to $T_{2}(L)=T_{2}(\infty)+a /(\ln L)^{2}$ and $T_{3}(\infty)=T_{3}(L)+a^{\prime} L^{-1 / \nu}$ with Potts exponent $\nu=5 / 6$ (cyan and red dashed lines). We find $T_{2}(\infty)=1.194 \pm 0.002$ and $T_{3}(\infty)=1.2022 \pm 0.0005$, and nonuniversal constants $a=0.21 \pm 0.05, a^{\prime}=1.00 \pm 0.16$. Shaded boxes denote the best estimate for thermodynamic transition temperatures with a confidence of 1 standard deviation. This demonstrates that the Potts transition at $T_{3}$ occurs above the nematic-KT transition at $T_{2}$, a conclusion also reached in the absence of the corrections to scaling shown in Fig. 7. 
future work should explore the regime of small $\lambda$ in more detail, since the splitting of $T_{3}$ and $T_{2}$ is potentially larger there, and one could observe them directly. Novel algorithmic advances will be required to probe this regime, as we expect the splitting to be observable only at system sizes larger than the ones probed in this work.

Still, we note that we cannot fully exclude a scenario with a single transition in the Potts universality class, but we consider this unlikely at finite $\lambda$ based on our MC results. In other words, our numerical analysis shows that assuming that the stiffness makes a universal jump corresponding to an unbinding of nematic $q_{\phi}= \pm 1$ vortices, the associated KT transition lies slightly below the Potts transition for all system sizes larger than $L=60$. Extrapolation to infinite system size leads to the conclusion that a gap fully opens between those two transitions' temperatures, revealing the region on LR Potts relative order. In contrast, a single transition is expected in the $\lambda \rightarrow \infty$ limit of our model (3.1), in analogy to the phase diagram obtained for the vector-nematic generalized XY model version of our model $[60,61,95]$, where it is argued that vortex unbinding is suppressed by critical fluctuations of the discrete order parameter. Further work, both numerical and analytical, should also be directed toward the $\lambda \rightarrow \infty$ limit of Eq. (3.8), especially in the region of $\Delta \simeq 1.0$, to explore its phase diagram further and test this conjecture.

To further study the nature of the phase transitions, we perform a finite-size-scaling analysis of specific heat $c$, Potts magnetization $m_{\sigma}$, and susceptibility $\chi_{\sigma}$. We find that the best data collapse after finite-size scaling of a given observable $\mathcal{O}$ is obtained using corrections to scaling $[128,131,132]$. The scaling for an observable $\mathcal{O}(t, L)$, with $t$ the reduced temperature and $L$ the system size, is given by the following form:

$$
\begin{aligned}
\tilde{t} & =L^{1 / \nu}\left(T-T_{c}\right) /\left(1+a_{2} L^{-\omega_{2}}\right), \\
\tilde{\mathcal{O}}(t, L) & =\frac{L^{\zeta / \nu}}{1+a_{1} L^{-\omega_{1}}} \mathcal{O}(\tilde{t}, L) .
\end{aligned}
$$

As shown in Fig. 7, a proper rescaling of the axes leads to a data collapse of results for different system sizes $40 \leq L \leq$ 380 and temperatures. The collapse is most complete for the magnetization $m_{\sigma}$ but also evident for $c$ and $\chi_{\sigma}$. The critical exponents presented in Table II that we extract from the scaling analysis are consistent with a transition in the Potts universality class. The scaling analysis yields a transition temperature of $T_{3}=1.20 \pm 0.01$ that agrees with the more precise value that is obtained from the scaling of the maximum of the specific heat with $L$, without corrections to scaling, as shown in Fig. 6(c). In essence, the addition of corrections to scaling does not change our estimate of $T_{3}(\infty)$, but it does provide valuable confirmation that this second-order upper transition truly lies in the Potts universality class. The temperature $T_{3}$ therefore (a)

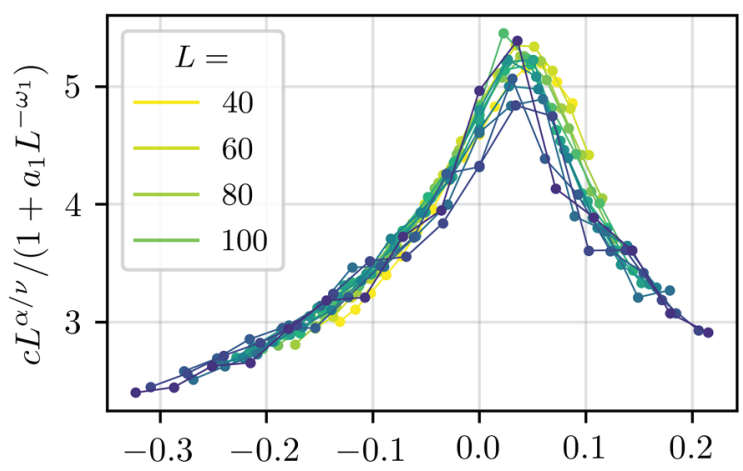

(b)

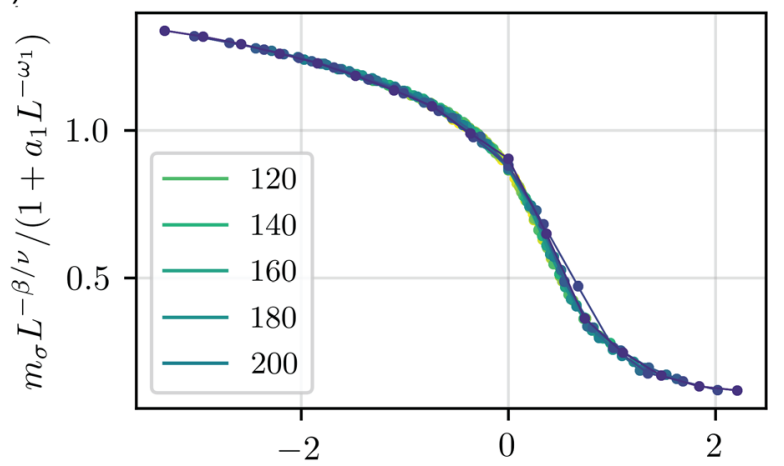

(c)

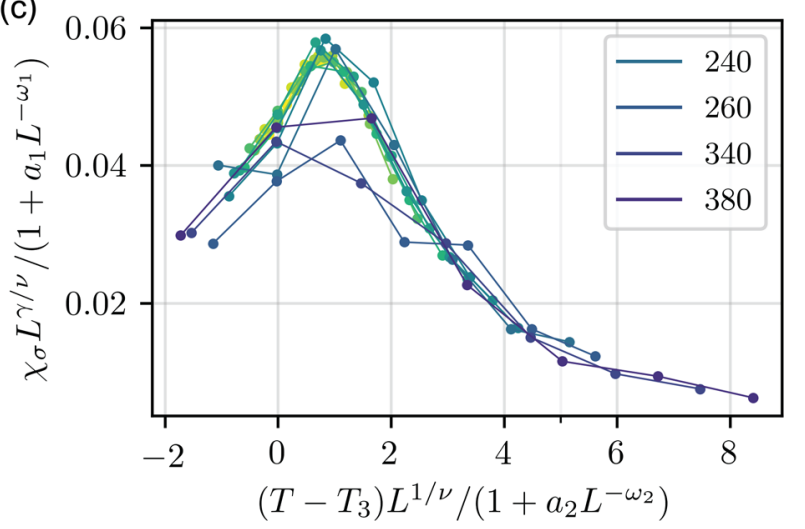

FIG. 7. Finite-size-scaling analysis of (a) specific heat $c$, (b) Potts magnetization $m_{\sigma}$, and Potts susceptibility $\chi_{\sigma}$ for system sizes between $L=40$ and $L=380$. We use the indicated scaling functions, as presented in Eq. (5.1), which correspond to a $2 \mathrm{D}$ continuous Potts transition and include corrections to scaling. The resulting critical exponents and the Potts transition temperature $T_{3}$ are collected in Table II. Data collapse is best for $m_{\sigma}$, but also works fairly well for $c$ and $\chi_{\sigma}$.

corresponds to the breaking of $\mathbb{Z}_{3}$ symmetry associated with the development of LR order in the relative orientation of hexatic and nematic angles.

\section{Single nematic-KT transition at $\Delta>\mathbf{1 . 1 5}$}

Finally, we briefly discuss the phase diagram at larger values of $1.15 \leq \Delta \leq 2$, where only a single nematic-KT transition occurs as a function of $T$ (see Fig. 4). We identify the location and nature of the phase transition by the 
TABLE II. Universal scaling exponents and critical temperature $T_{c}$ extracted from finite-size-scaling analysis of $c, m_{\sigma}$, and $\chi_{\sigma}$ shown in Fig. 7. We use the scaling form presented in Eq. (5.1), which includes corrections to scaling. The $2 \mathrm{D}$ Potts critical exponents are $\alpha=1 / 3, \beta=1 / 9 \approx 0.11, \gamma=13 / 9 \approx 1.44$, and $\nu=5 / 6 \approx 0.83$, which is in fair agreement with our findings with the exception of $\nu$. Note that when tracking only $T_{3}$, and including small system sizes, we obtain a value of $\nu$ that lies much closer to its expected value, a conclusion [Fig. 6(a)].

\begin{tabular}{lccccccl}
\hline \hline $\mathcal{O}$ & $T_{c}$ & $\zeta$ & $\nu$ & $a_{1}$ & $\omega_{1}$ & $a_{2}$ & $\omega_{2}$ \\
\hline$c$ & 1.20 & $\alpha=0.30$ & 1.45 & 10 & 1.1 & 100 & 0.008 \\
$m_{\sigma}$ & 1.20 & $-\beta=-0.18$ & 1.25 & 0.15 & 0.13 & 0.15 & 0.19 \\
$\chi_{\sigma}$ & 1.20 & $\gamma=1.44$ & 0.95 & 0.93 & 0.11 & 0.1 & 0.05 \\
\hline \hline
\end{tabular}

universal jump criterion of the stiffness $\rho\left(T_{2}\right)=2 T_{2} / \pi$ and find that the jump corresponds to an unbinding of nematic vortices of charge $q_{\phi}=1$. Because of the locking constraint (3.4), these are equivalent to $q_{\theta}=3$ hexatic vortices. Again, by fractionalizing the nematic vortices inside an extended vortex core so that there are three $q_{\theta}=1$ vortices, the energy of such a composite vortex can be lowered, especially at smaller values of $\lambda$. As $T_{2}<T_{\lambda}$, QLRO of the nematic degrees of freedom immediately leads to QLRO of

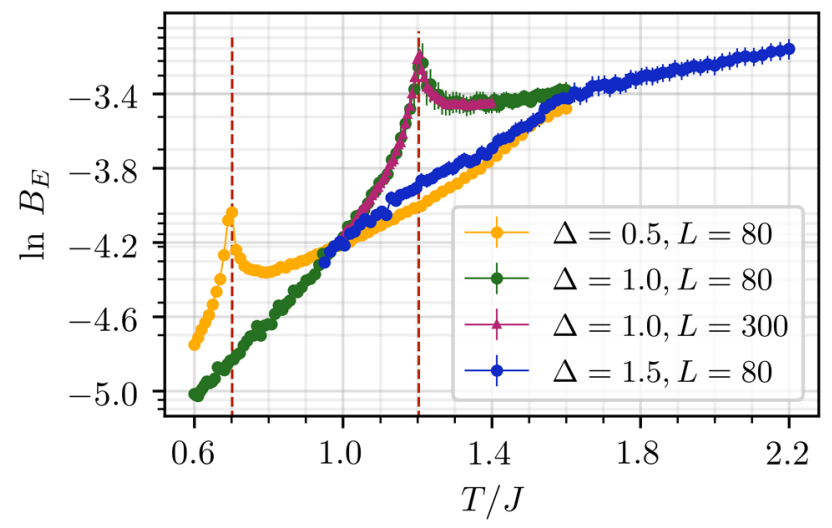

FIG. 8. Binder cumulant of the energy $B_{E}=\left(\left\langle E^{4}\right\rangle /\left\langle E^{2}\right\rangle^{2}\right)-1$ as a function of the temperature $T / J$ for different values of $\Delta$. The system size is set to $L=80$. At $\Delta=0.5$, the energy Binder develops a sharp peak at the Potts transition $T_{3}$ (vertical dashed line) as obtained in Fig. 4(b). The apparent change in slope at around $T=1.5 \mathrm{~J}$ coincides with the characteristic broad hump in the specific heat at around $1.1 T_{6}=1.5 \mathrm{~J}$ with $T_{6}=1.35 \mathrm{~J}$. At $\Delta=1.0$, the sharp peak associated with the Potts transition has moved up in temperature and is located at $T_{3}$ that we extract most precisely from the specific heat (Fig. 6). The presence of a sharp peak, which persists up to the largest system sizes we consider, $L=300$, is a clear indication of a Potts transition at $\Delta=1.0$. Note that since $B_{E} \propto L^{-2}$, we rescale the $L=300$ curve (magenta triangles) by $(300 / 80)^{2}$, which makes it lie on top of the $L=80$ curve (green circles). In contrast, such a sharp peak is notably absent at $\Delta=1.5$, where one observes only a change in slope around $T=1.6 \mathrm{~J}$ associated with the single nematic-KT transition at $T_{2}$. the hexatic variables, as they feel a uniaxial potential with a single minimum. Both $\mathrm{O}(2)$ and $\mathbb{Z}_{3}$ symmetries are thus broken at the nematic-KT transition for $\Delta>1.15$. This is confirmed clearly by the Binder cumulants $B_{\theta}, B_{\phi}, B_{\sigma}$ at $\Delta=1.5$, which are shown in the right column of Fig. 10 in Appendix C. Both $B_{\phi}$ and $B_{\sigma}$ undergo a sharp transition at $T_{2}$, while $T_{6}$ exhibits a smoother crossover toward the value of 2/3 consistent with QLRO as the temperature is lowered. As shown in Fig. 8, the Binder cumulant of the energy $B_{E}$ shows only a change in slope above $T_{2}$ and does not exhibit any additional feature at temperatures $T<T_{2}$.

Finally, the specific heat exhibits two (broad) maxima. The first, at a temperature above $T_{2}$, corresponds to the usual broad KT maximum at $1.1 T_{2}$, where nematic vortices proliferate. In addition, a maximum appears below $T_{2}$ that roughly follows the hexatic phase transition in the uncoupled system at $\lambda=0$ (Fig. 3). This crossover is due to the production of $q_{\theta}=1$ hexatic vortices that are bound to fractionalized and confined $q_{\phi}=1 / 3$ vortices. These hexatic vortices are thus bound together by domainwall strings of finite tension and release only a small part of the entropy, leading to a less pronounced maximum of $c$. These topological defects do not lead to a jump of the total stiffness, rather to a gradual decrease.

\section{CONCLUSION AND OUTLOOK}

Motivated by a long-standing experimental mystery in liquid-crystal films of 54COOBC, we have presented a detailed Monte Carlo simulation study of a generalized $\mathrm{XY}$ model with finite coupling strength $\lambda$ between a hexatic and nematic degree of freedom. Our key finding is that there exists a parameter region in the phase diagram [for $\Delta \approx 1$ in Eq. (3.1)], where a sharp specific heat signature occurs at a temperature $\left(T_{3}\right)$ above the temperature where the spin stiffness jumps to its finite value; all thermodynamic exponents are consistent with $\mathbb{Z}_{3}$ symmetry. This phase has relative nematic-hexatic order and can be characterized as one of free $q_{\phi}=1$ nematic vortices coupled to three bound $q_{\theta}=1$ hexatic vortices. These composite nematic vortices are shown schematically in Fig. 1(d). At lower temperatures, our numerical scaling analysis demonstrates the presence of a Kosterlitz-Thouless transition $\left(T_{\mathrm{KT}, 2}\right.$ or $\left.T_{\mathrm{KT}, \mathrm{nem}}\right)$, where composite nematic-hexatic vortices become bound. For the coupling value $\lambda=2.1$ chosen to benchmark with past studies, in our simulations we find the ratio of the transition temperature scales to be $T_{3} / T_{\mathrm{KT}, 2}=1.007$. Both nematic and hexatic degrees of freedom develop only quasi-longrange order below $T_{\mathrm{KT}, 2}$. Our simulations show no signs of first-order behavior anywhere in the phase diagram.

This scenario closely resembles the experimental observations in single-layer films of 54COOBC with a sharp specific heat signature between two smectic phases ( $\mathrm{Sm}-A$ to $\left.\mathrm{Sm}-A^{\prime}\right)$ at $T_{\mathrm{Sm}-A^{\prime}}=66^{\circ} \mathrm{C}$ and a lower KT transition into a hexatic phase (Sm- $A^{\prime}$ to Hex- $B$ ) at $T_{\mathrm{Hex}-B}=63^{\circ} \mathrm{C}$. The ratio 
of experimental transition temperatures is given by $T_{\mathrm{Sm}-A^{\prime}} / T_{\text {Hex- } B}=1.018$, consistent with our findings. The transition into the $\mathrm{Sm}-A^{\prime}$ phase is characterized by a sharp specific heat divergence with $\alpha \approx 0.3$, in agreement with the 2D Potts exponent $\alpha=1 / 3$ that we obtain in our investigation of this minimal model. While experimentally the hexatic correlation length makes a sudden jump into the Sm$A^{\prime}$ phase, it remains finite, and hexatic QLR order develops only at $T_{\mathrm{Hex}-B}$, again resembling our simulation results of a lower KT transition.

We note that, in other freestanding thin films such as the $n m$-OBC family, the hexatic phase observed is Hex- $E$ rather than Hex- $B$. In contrast to the Hex- $B$ phase, for Hex- $E$ there appear additional satellite peaks in the scattering pattern associated with (nematic) herringbone order [85]. This is likely due to a strong packing of the molecules in these compounds [80,81]. It is possible that $54 \mathrm{COOBC}$ is not as strongly packed and that the microscopic van der Walls interactions between the nematic molecules lead to more complex local ordering which does not lead to such satellite peaks. We hope that these liquid-crystal-specific issues that we bring to light here stimulate further research on the microscopic origin of the local nematic order in 54COOBC.

There are several additional features that emerge from our study. For example, we reproduce previously reported results at smaller values of $\Delta$ [34], where a Potts transition occurs below a hexatic-KT transition $T_{3}<T_{\mathrm{KT}, 6}\left(=T_{\mathrm{KT} \text {,hex }}\right)$ that is characterized by the unbinding $q_{\theta}=1$ vortices. Each hexatic defect is attached to a Potts domain wall [Fig. 1(a)] so that the binding of these defects is accompanied by the formation of a network of local Potts domains. The prior establishment of a Potts transition at lower temperatures can be understood as the vanishing of these Potts walls and the formation of a single Potts domain.

Our analytic estimates suggest that confinement and vortex fractionalization play important roles in the emergence of the composite Potts phase. At a temperature $T_{\text {conf }}>T_{\mathrm{KT}, 2}, q_{\phi}=1$ vortices form composed of bound states of three $q_{\theta}=1$ hexatic defects [Fig. 1(d)]. Again, the absence of "dangling" Potts walls means that a network of local Potts domains is formed. Then, the question is whether the Potts ordering $T_{3}$ is above or at $T_{\mathrm{KT}, 2}$ since there can be no Potts domain walls once the $q_{\phi}$ defects bind. Our extensive numerical MC simulations show that $T_{3}>T_{\mathrm{KT}}$ in the region $0.9<\Delta<1.15$. This suggests that the emergence of extended vortex core sizes formed of bound fractional vortices lower the KT transition to a value below $T_{3}$, revealing a new phase.

The confinement of fractional vortices could, in principle, occur in other coupled XY models of competing periodicities, where the $\lambda$ term locks the fields. This locking changes the overall symmetry from $\mathrm{U}(1) \times \mathrm{U}(1)$ for the decoupled case to $\mathrm{U}(1) \times \mathbb{Z}_{p}$ ( $p=3$ corresponds to this work), where the $\mathbb{Z}_{p}$ symmetry is the relative ordering of the fields. Only $p \leq 4$ may exhibit a second-order phase transition above a KT transition [76]. Whereas we present here a liquid-crystal setting of a $p=3$ model, the specific case of $p=2$ is directly related to atom-molecular mixtures $[6,7]$. We hope that our work may provide an impetus to future numerical and analytical studies of coupled $\mathrm{XY}$ models, which might reveal related hidden relative phases.

In order to maintain continuity and consistency with previous computational studies, we explore the hexaticnematic XY model [Eq. (3.1)] with the same coupling value $(\lambda=2.1)$ as before [34]. Since the vortex core size increases with decreasing hexatic-nematic coupling strength $\lambda$, we expect that the two temperature scales $T_{3}$ and $T_{\mathrm{KT}, 2}$ will be further separated at smaller values of $\lambda$, which should be explored in the future. Significantly larger system sizes will, however, need to be simulated for smaller $\lambda$, as the composite vortices' extent is expected to increase. This should be accessible using techniques such as the worm algorithm [60,61,133] and matrix-product states [98]. Future studies of the effect of tuning $\lambda$ on the ratio of temperatures $T_{3} / T_{\mathrm{KT}, 2}$ are therefore desirable to probe why the observed ratio for our study is so small, even if it is aligned with the experimental feature.

An alternative tuning parameter is hexatic ring-exchange coupling [74,134-137] that is known to tune vortex core energies and could thus be used to suppress the lower KT transition, expanding the Potts phase. Tuning this parameter is straightforward in numerical simulations. It has also been shown experimentally that core energies and sizes are correlated with liquid-crystal densities [74], providing an experimental tuning parameter that could lead to a larger $\mathrm{Sm}-A^{\prime}$ phase region. Adjusting these parameters may be done by varying local density, possibly by bending the freely suspended liquid-crystal films of 54COOBC. Because the proposed Potts phase involves relative hexatic-nematic ordering and hence higher-order correlations, it is challenging to probe these composite order parameters experimentally since most probes measure two-point correlation functions of the primary orders.

Taking our cue from intriguing results in a related model $[60,61,97]$, it is an open question whether the two temperature scales $T_{3}$ and $T_{\mathrm{KT}, 2}$ converge into a single transition at large values of $\lambda$. The two end points in $\Delta$ of our composite Potts relative phase are worth further study. At $\Delta \in\{0.85,0.9\}$, the possible existence of a multicritical fixed point of $\mathrm{O}(2) \times \mathbb{Z}_{3}$ symmetry suggests the exciting possibility of an emergent supersymmetry [117], in analogy to the FFXY model [89]. Close to $\Delta=1.15$, where the Potts transition disappears, there may be a deconfined critical point $[60,61,97]$.

In conclusion, we demonstrated a new sequence of phase transitions in coupled XY models characterized by an intermediate phase with relative discrete order hosting extended composite topological defects. We have presented the 54COOBC liquid-crystal film problem as an experimental realization of the deconfinement of fractional vortices; here the "mystery" phase above the nematic-KT transition is one of 
composite Potts ordering in the relative hexatic-nematic angles. We have reproduced the observed specific heat behavior as a function of the temperature in our computational study of a minimalist model. The emergent Potts phase hosts extended nematic vortices that are analogous to baryons. Furthering the analogy, the three hexatic vortices that form such a bound state are akin to quarks. From this perspective, the experimentally observed Potts transition in the liquidcrystal samples is a laboratory realization of quark confinement and baryon formation. Of course, this is an Abelian theory, whereas QCD is, of course, non-Abelian. Nevertheless, we think the analogy stands and that one can gain valuable insight into the issue of confinement of fractional objects in 2D.

Although we have focused on a liquid-crystal setting to motivate our study, we expect that very similar physics can be at play in atom-molecular mixtures of Bose-Einstein condensates or low-dimensional superconductors. Our work raises several further questions regarding the deconfinement of fractional vortices, in particular, the need for a fully developed analytic theory for the interaction of domain walls with fractional vortices. We hope that our results will stimulate further interest in these coupled XY problems and their physical realizations.

\section{ACKNOWLEDGMENTS}

We thank Kun Chen, Paul Fendley, Ludovic Jaubert, Elio J. König, Leo Radzihovsky, Subir Sachdev, Jörg Schmalian, and A. Peter Young for useful discussions, and we are grateful to David Vanderbilt for providing us access to the Beowulf cluster at Rutgers University. This work was supported by the U.S. Department of Energy (DOE), Office of Science, Basic Energy Sciences under Awards No. DESC0020353 (P. Chandra) and No. DE-FG02-99ER45790 (P. Coleman and V. D. T.), as well as by the National Science Foundation under Grant No. NSF MRSEC/DMR-1720530 (T. C. L.). V. D. T. also acknowledges the support of the Fonds de Recherche Québécois en Nature et Technologie. Part of the research (P. P. O.) was performed at the Ames Laboratory, which is operated for the U.S. DOE by Iowa State University under Contract No. DE-AC02-07CH11358.

\section{APPENDIX A: RELEVANCE OF HEXATIC-NEMATIC COUPLING}

We explore the relevance of the hexatic-nematic coupling $\propto \lambda \cos [6(\tilde{\vartheta}-\tilde{\varphi})]$ in Eq. (2.4). The intermediate numerical factors are different for the models of Eqs. (2.1) and (3.1), but the end result is identical. We first turn to a long-wavelength version of the model

$\tilde{\mathcal{H}}=\int d^{2} r\left[\frac{K_{6}^{\prime}}{2}(\nabla \tilde{\vartheta})^{2}+\frac{K_{2}^{\prime}}{2}(\nabla \tilde{\varphi})^{2}-\lambda_{3} \cos [6(\tilde{\vartheta}-\tilde{\varphi})]\right]$.
For $K_{2}^{\prime}=K_{6}^{\prime}$ [i.e., $\Delta=1.0$ in Eq. (3.1)], one has that the hexatic $T_{\mathrm{KT}, 6}$ and nematic $T_{\mathrm{KT}, 2}$ are at identical temperatures, i.e., $T_{\mathrm{KT}, 6}=T_{\mathrm{KT}, 2}$. We can find whether the coupling constant $\lambda_{3}=\tilde{\lambda} / T$ is a relevant perturbation at the KT transition temperature by using the fact that at the transition $T_{\mathrm{KT}, p}$ the exact value of the renormalized stiffnesses is known to be

$$
K_{R, p}^{\prime}\left(T_{\mathrm{KT}, p}\right)=\frac{2 p^{2}}{\pi},
$$

with bare $K_{p}^{\prime}=J_{p} / T$ from the model presented in Eq. (2.4). Following Refs. [17,138], we evaluate the correlation function associated with the coupling between $\vartheta$ and $\varphi$, assuming no correlations between $\tilde{\vartheta}$ and $\tilde{\varphi}$, i.e., $\left\langle[\tilde{\vartheta}(x)-\tilde{\varphi}(0)]^{2}\right\rangle=0$, valid for $\lambda_{3}=0$ via calculating

$$
\begin{aligned}
\mathcal{C}_{\lambda}(x) & =\left\langle e^{6 i[\tilde{\vartheta}(x)-\tilde{\vartheta}(0)-\tilde{\varphi}(x)+\tilde{\varphi}(0)]\rangle}\right. \\
& =e^{-\frac{36}{2}\left[(\tilde{\vartheta}(x)-\tilde{\vartheta}(0)]^{2}\right\rangle-\frac{36}{2}\left[(\tilde{\varphi}(x)-\tilde{\varphi}(0)]^{2}\right\rangle} \\
& =e^{-\frac{36}{2 \pi}\left(\frac{1}{K_{R, 6}^{\prime}}+\frac{1}{K_{R, 2}^{\prime}}\right) \ln \frac{x}{a_{0}}} \\
& =\left|\frac{x}{a_{0}}\right|^{-\eta_{c}},
\end{aligned}
$$

where we use $\frac{1}{2}\left\langle[\psi(x)-\psi(0)]^{2}\right\rangle \approx[1 /(2 \pi K)] \ln \left|x / a_{0}\right|$ for Gaussian field $\psi$ with coupling $K$. The exponent is

$$
\eta_{c}=\frac{18}{\pi}\left(\frac{1}{K_{R, 6}^{\prime}}+\frac{1}{K_{R, 2}^{\prime}}\right) .
$$

Via the Kadanoff construction $[17,138]$, we find whether $\lambda_{3}$ is a relevant perturbation by determining the sign of the scaling dimension

$$
\mathcal{D}_{\lambda_{3}}=2-\frac{\eta_{c}}{2}=2-\frac{9}{\pi}\left(\frac{1}{K_{R, 6}^{\prime}}+\frac{1}{K_{R, 2}^{\prime}}\right) .
$$

Using Eq. (A2) at $T_{\mathrm{KT}, 6}=T_{\mathrm{KT}, 2}$, we obtain

$$
\left.\mathcal{D}_{\lambda_{3}}\right|_{T=T_{\mathrm{KT}, 6}=T_{\mathrm{KT}, 2}}=\frac{3}{4}>0 .
$$

This means that $\lambda_{3}$ is a relevant perturbation at $T=T_{\mathrm{KT}, 6}=T_{\mathrm{KT}, 2}$, and the system will flow away from the KT transition at nonzero $\lambda_{3}$, and hence, the system will encounter a high-temperature $T_{\lambda}$ scale where the variables become locked with one another, satisfying the $\lambda$ coupling. This completes the derivation of the result presented at Eq. (3.3) obtained for model (3.1) but otherwise identical.

\section{APPENDIX B: ESTIMATION OF THE SIZE OF COMPOSITE VORTICES}

In this section, we provide a rough estimate of the size of a composite vortex of total charge $q_{\phi}=1$ formed of three hexatic vortices $\left(q_{\theta}=1\right)$ bound through a domain wall of 
the $\sigma$ variable, such that $\sigma \rightarrow \sigma+2 \pi / 3$ across such a wall. This procedure was done in Refs. $[8,59,139]$ with relevance to quark deconfinement and in Ref. [6] for the $\mathbb{Z}_{2}$ symmetric case. We largely reproduce the treatment of this last reference here for our $\mathbb{Z}_{3}$ symmetric case.

We consider an effective long-wavelength model analogous to Eq. (3.1) obtained by transforming $\cos \left(\theta_{i}-\theta_{j}\right) \sim$ $d r(\nabla \theta)^{2} / 2$ into the continuum limit with $K_{2}=\Delta / T$, $K_{6}=(2-\Delta) / T$, and $h=\lambda / T$ :

$\bar{H}=\int d^{2} r\left[\frac{K_{6}}{2}(\nabla \theta)^{2}+\frac{K_{2}}{2}(\nabla \phi)^{2}-h \cos (\theta-3 \phi)\right]$.

Note that one can relate $K_{2}$ and $K_{6}$ to those of the original model of Eq. (2.4) used in Appendix A, such that $K_{p}=p^{2} K_{p}^{\prime}$. We consider two types of point defects in the two $\mathrm{XY}$ variables:

$$
\oint_{r_{0}} \nabla \theta(\mathbf{r}) \cdot d \mathbf{l}=2 \pi q_{\theta}, \quad \oint_{r_{0}} \nabla \phi(\mathbf{r}) \cdot d \mathbf{l}=2 \pi q_{\phi} .
$$

It is clear that the two variables have their own independent behavior for $h=0$. However, for any finite $h$, an extensive energy cost is incurred if the system does not lock the two phases on average with each other such that $\langle\theta\rangle=3\langle\phi\rangle$. This further leads to the winding numbers being related to each other, as we explain in Eq. (3.7). The first way to satisfy this is to simply have $q_{\phi}=1$ and $q_{\theta}=3$. However, as we show later, this is very costly as the phase has to wind very tightly around the vortex, generating a large core energy for the vortex. The second way to satisfy this constraint is to have $q_{\theta}=1$ and $\Delta n=1$ (or equivalently, $\left.q_{\phi}=1 / 3\right)$. In the simplest picture, this fractional vortex in the $\phi$ generates a line defect originating at the vortex core [Fig. 9], through which, $\phi$ will rapidly wind by $2 \pi / 3$. It is possible, however, that some part of the rapid winding "leaks" into the $\theta$ variable. For $h$ extremely large or $K_{i}$ very small, we expect that this wall defect will be very thin $\left(\xi_{\mathrm{DW}} \ll \xi_{i}\right)$, but as $h$ is decreased, it should become wider and wider, leading to a more gradual domain wall in $\phi$. Note that in both cases, the energy of such a wall will scale linearly with the length of the wall, such that $E_{\mathrm{DW}} \propto L$.

These walls separate domains of different $n=-1,0,1$ where we have $2 \pi n / 3=\sigma=\frac{1}{3}(\theta-3 \phi)$ following Eq. (4.4). One can clearly isolate a solution for the domain wall by rewriting the phase variables

$$
\begin{gathered}
\alpha=\frac{\theta+3 \phi}{2}, \quad \sigma=\frac{\theta-3 \phi}{3}, \\
\text { or } \quad \theta=\alpha+\frac{3 \sigma}{2}, \quad \phi=\frac{\alpha}{3}-\frac{\sigma}{2} .
\end{gathered}
$$

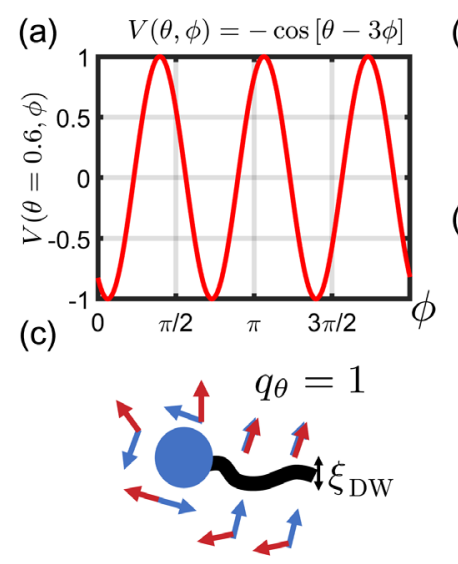

(b)

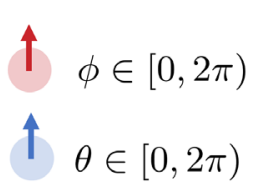

(d) $q_{\phi}=1$ with radius $R$

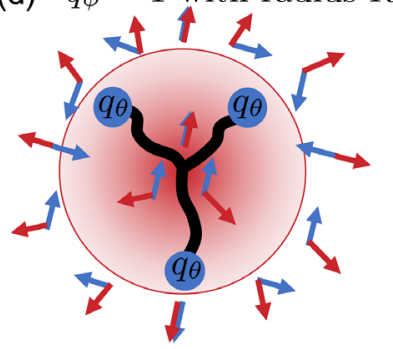

FIG. 9. (a) Illustration of the $\lambda$ constraint; domain walls of the relative variable $\sigma=2 \pi n / 3$, as defined in Eq. (4.4), are situations where the $\theta$ and $\phi$ do not fall in the deep minima of $V$. (b) The domain of both $\theta$ and $\phi$ is $[0,2 \pi)$ corresponding to the model of Eq. (3.1). This is different from the $\vartheta$ and $\varphi$ variables from Fig. 1 that are defined $\bmod 2 \pi / 3$ and $2 \pi$, respectively. (c) Visualization of a hexatic vortex $q_{\theta}=1$ with its adjacent domain wall of the relative variable $\sigma=2 \pi n / 3$ of width $\xi_{\mathrm{DW}}$. This can also be viewed as a fractional $q_{\phi}=\frac{1}{3}$ vortex. (d) A composite vortex of total charge $q_{\phi}=1$ and $q_{\theta}=3$, which is clearly seen outside the core. It is formed of three $q_{\theta}=1$ vortices [blue dots; see panel (c)] behaving as fractional $q_{\phi}=1 / 3$ objects bound by their domain-wall strings. These fractional objects are arranged in this simple geometry where the walls are of length $R$ and the distance between $q_{\theta}=1$ vortices is $\sqrt{3} R$.

This transformation is valid as long as the phases are well defined. The model of Eq (B1) is rewritten as

$$
\begin{aligned}
\bar{H}= & \int d^{2} r\left[\frac{K_{a}}{2}(\nabla \alpha)^{2}+\frac{K_{b}}{2}(\nabla \sigma)^{2}\right. \\
& \left.-K_{a b}(\nabla \alpha) \cdot(\nabla \sigma)-h \cos (3 \sigma)\right]
\end{aligned}
$$

with

$$
\begin{aligned}
K_{a} & =\left(K_{6}+K_{2} / 9\right), \\
K_{b} & =\left(9 K_{6}+K_{2}\right) / 4, \\
K_{a b} & =\frac{1}{2}\left(K_{2} / 3-3 K_{6}\right) .
\end{aligned}
$$

Understanding that this Hamiltonian is simply $\bar{H}=$ $\int d^{2} r \mathcal{E}(\alpha(r), \sigma(r))$ with $\mathcal{E}$ the energy density, and we write the saddle-point equations $\delta \mathcal{E} / \delta \alpha=\delta \mathcal{E} / \delta \sigma=0$, which leads to

$$
\begin{gathered}
-K_{a} \nabla^{2} \alpha+K_{a b} \nabla^{2} \sigma=0, \\
-K_{b} \nabla^{2} \sigma+K_{a b} \nabla^{2} \alpha+3 h \sin (3 \sigma)=0 .
\end{gathered}
$$


We can eliminate $\alpha$ from these equations and find the known sine-Gordon equation for the $\sigma$ variable

$$
\begin{aligned}
& -K \nabla^{2} \sigma+3 h \sin (3 \sigma)=0 \\
& \text { with } \quad K=K_{b}-K_{a b}^{2} / K_{a} .
\end{aligned}
$$

We solve this equation for the situation where we have $\sigma(x, y \rightarrow \infty) \rightarrow 0$ and $\sigma(x, y \rightarrow-\infty) \rightarrow 2 \pi / 3$ (this is done without loss of generality, as all domain walls have a jump of $2 \pi / 3$ ), which leads to a domain wall along the $x$ direction. The solution for this domain wall is then simply

$$
\begin{aligned}
\sigma_{\mathrm{DW}}(y) & =\frac{4}{3} \arctan \left(e^{2 y / \xi_{\mathrm{DW}}}\right) \\
\text { with } \xi_{\mathrm{DW}} & =\frac{2}{3} \sqrt{\frac{2 K}{3 h}} .
\end{aligned}
$$

We get that $\alpha_{\mathrm{DW}}(y)=\left[\left(K_{a b}\right) / K_{a}\right] \sigma_{\mathrm{DW}}(y)$ and then, for our initial variables

$$
\begin{aligned}
\theta_{\mathrm{DW}} & =\left(\frac{3}{2}+\frac{K_{a b}}{K_{a}}\right) \sigma_{\mathrm{DW}}(y), \\
\phi_{\mathrm{DW}} & =\frac{1}{2}\left(\frac{2 K_{a b}}{3 K_{a}}-1\right) \sigma_{\mathrm{DW}}(y) .
\end{aligned}
$$

One has that the energy of a domain wall of length $L$ is $E_{\mathrm{DW}}=\epsilon_{\mathrm{DW}} L$ with

$$
\begin{aligned}
\epsilon_{\mathrm{DW}}= & \int d y\left[\frac{K_{a}}{2}\left(\nabla \alpha_{\mathrm{DW}}\right)^{2}+\frac{K_{b}}{2}\left(\nabla \sigma_{\mathrm{DW}}\right)^{2}\right. \\
& \left.-K_{a b}\left(\nabla \alpha_{\mathrm{DW}}\right) \cdot\left(\nabla \sigma_{\mathrm{DW}}\right)-h \cos \left(3 \sigma_{\mathrm{DW}}\right)\right] \\
= & \int d y\left[\frac{K}{2}\left(\nabla \sigma_{\mathrm{DW}}\right)^{2}-h \cos \left(3 \sigma_{\mathrm{DW}}\right)\right] \\
= & \int d y\left[\frac{K}{2}\left(\nabla \sigma_{\mathrm{DW}}\right)^{2}+\frac{K}{2}\left(\nabla \sigma_{\mathrm{DW}}\right)^{2}\right] \\
= & \int d y K\left(\nabla \sigma_{\mathrm{DW}}\right)^{2}=\frac{16 K}{9 \xi_{\mathrm{DW}}}=4 \sqrt{\frac{2 K h}{3}} .
\end{aligned}
$$

There is a caveat to this result. As $K \rightarrow 0$, the energy of the wall goes to 0 and so does its width. On the other hand, as $h \rightarrow \infty$, the wall width goes to 0 , but the energy increases dramatically. It is important in this context to bring back the lattice cutoff $a \sim 1$ here. Hence, we have $\xi_{\mathrm{DW}}=\frac{2}{3} \sqrt{[(2 K) /(3 h)}>1$. For $h<4 K / 9$, the theory is well behaved. For larger $h$, one has to use $\xi_{\mathrm{DW}}=1$ and adjust Eq. (B18). Note that putting back the initial parameters $K_{2}, K_{6}$, and $h$ in the domain-wall energy we get $K=9\left(K_{2} K_{6}\right) /\left(K_{2}+9 K_{6}\right)$, which leads to the following expression for the domain-wall energy:

$$
\epsilon_{\mathrm{DW}}=4 \sqrt{6 h\left(\frac{K_{2} K_{6}}{K_{2}+9 K_{6}}\right)},
$$

or, using $\Delta$,

$$
\epsilon_{\mathrm{DW}}=4 \sqrt{3 h\left(\frac{\Delta(2-\Delta)}{9-4 \Delta}\right)} .
$$

With this new insight, we wish to compare the energy of a point defect with $q_{\phi}=1$ to the assembly of three hexatic objects with each $q_{\theta}=1$ linked by a domain wall for the same total charge. This arrangement is shown in Fig. 9. We denote the first situation as a point vortex (PV) and the second as a split vortex (SV). The energy of a pure defect with no domain wall, i.e., one in which a $q_{\theta}=3$ vortex is accompanied by a $q_{\phi}=1$ vortex, is simply expressed as

$E_{\mathrm{PV}}=E_{c, \theta}(3)+E_{c, \phi}(1)+9 \pi K_{6} \ln \left(L / \xi_{6}\right)+\pi K_{2} \ln \left(L / \xi_{2}\right)$

$$
\begin{aligned}
= & {\left[\frac{\pi^{2}}{2}\left(9 K_{6}+K_{2}\right)+9 \pi K_{6} \ln \left(R / \xi_{6}\right)+\pi K_{2} \ln \left(R / \xi_{2}\right)\right] } \\
& +9 \pi K_{6} \ln (L / R)+\pi K_{2} \ln (L / R) \\
= & E_{\mathrm{PV}}^{c}(R)+9 \pi K_{6} \ln (L / R)+\pi K_{2} \ln (L / R) .
\end{aligned}
$$

The second line uses the first approximation to the core energy of the vortices $E_{c} \sim \pi^{2} q^{2} K / 2$, with $q$ being the charge of the vortex. Note that in this formula, we explicitly introduce an arbitrary radius $R$ and split the log parts. This comes in handy when we compare this new core energy $E_{\mathrm{PV}}^{c}(R)$ to the split core energy $E_{\mathrm{SV}}^{c}(R)$, as we consider $R$ to be the radial length of a split combination of vortices. We are then able to compare only the energetics inside the radius $R$, since for $r>R$, both combinations will look and act like a point vortex. This point vortex is thought to be the dominant kind as $h \gg K$, since then the domain-wall width becomes extremely small and its energy very large.

In the case of a split vortex, we need to include some extra energetic terms, i.e., the logarithmic repulsion between the vortices themselves $V_{q-q}(r)=-2 \pi K q^{2} \ln (r / \xi)$. There are three such terms here for each type of vortex, and they are separated by a distance of $\sqrt{3} R$ for this simple geometry (Fig. 9). We also include the attractive domain-wall energy $\epsilon_{\mathrm{DW}} R$ for each of the domain walls. We then get

$$
\begin{aligned}
E_{\mathrm{SV}}^{c}(R)= & 3\left[E_{c, \theta}(1)+E_{c, \phi}(1 / 3)\right]-3 \times 2 \pi K_{6} \ln \left(\sqrt{3} R / \xi_{6}\right) \\
& -3 \times \frac{2 \pi}{9} K_{2} \ln \left(\sqrt{3} R / \xi_{2}\right)+3 R \epsilon_{\mathrm{DW}} \\
= & \frac{3 \pi^{2}}{2}\left(K_{6}+K_{2} / 9\right)-6 \pi K_{6} \ln \left(\sqrt{3} R / \xi_{6}\right) \\
& -\frac{2 \pi}{3} K_{2} \ln \left(\sqrt{3} R / \xi_{2}\right)+3 R \epsilon_{\mathrm{DW}} .
\end{aligned}
$$


At this point, we stress that point configurations, the point vortex and the split vortex, are topologically equivalent at $r>R$. We can then compare the two configurations in order to determine the region in which a split vortex would be less energetic than the point vortex.

We start by finding out the optimal size of a split vortex by finding $R_{\max }$ such that $\left.\left\{\left[\partial E_{\mathrm{SV}}^{c}(R)\right] /(\partial R)\right\}\right|_{R_{\max }}=0$. In the following, we use $\xi_{6}=\xi_{2}=a$. This leads to the following expression for the optimal radial size of a split vortex:

$$
R_{\max } / a=2 \pi \frac{K_{2} / 9+K_{6}}{\sqrt{3} \epsilon_{\mathrm{DW}}} .
$$

One sees that driving the coupling $h$ to infinity completely annihilates the split vortices' structures (by $\epsilon_{\mathrm{DW}} \rightarrow 0$ ). There is, however, a regime with low $h$ where the split vortex will be quite extended. Note that this expression does not depend on the temperature but only on the interaction parameters themselves.

In the case of strong coupling, i.e., $h>4 K / 9$ (for $K_{2}=K_{6}=1$, this is $h>4 / 10$ ), the domain-wall width is $\xi_{\mathrm{DW}}=a=1$, i.e., the lattice spacing. This is the case for the $\lambda=2.1$ that we choose for our simulations. In this case, we get

$$
\sigma_{\mathrm{DW}}(y)=\frac{4}{3} \arctan \left(e^{2 y}\right),
$$

which then leads to

$$
\epsilon_{\mathrm{DW}}=16 K / 9,
$$

and then, for $\Delta=1.0$, our computational value, we have

$$
R_{\max } / a \simeq 2.5,
$$

which completes the derivation of the vortex size estimate that is presented in Sec. III C. Using this value for the radius of the composite vortex into the energy estimates from Eqs. (B23) and (B25), we get that for such an extended vortex,

$$
\begin{aligned}
& E_{\mathrm{PV}}^{c}\left(R_{\max }\right) \simeq 78.13, \\
& E_{\mathrm{SV}}^{c}\left(R_{\max }\right) \simeq 5.98,
\end{aligned}
$$

proving that in the regime of our $\mathbb{Z}_{3}$ relative order phase, composite vortices are favored with respect to point ones due to their extended nature, even with the cost of domain walls in the core of the vortex.

\section{APPENDIX C: COMPARISON OF THE BINDER CUMULANT FOR DIFFERENT REGIMES}

We present here a comparison of the Binder cumulant for $M_{a}$ with $a=\theta, \phi, \sigma$ computed from Eq. (4.5c). This is shown in Fig. 10. The Binder cumulants approach a value of $1 / 3(2 / 3)$ in the disordered (ordered) state at high (low)
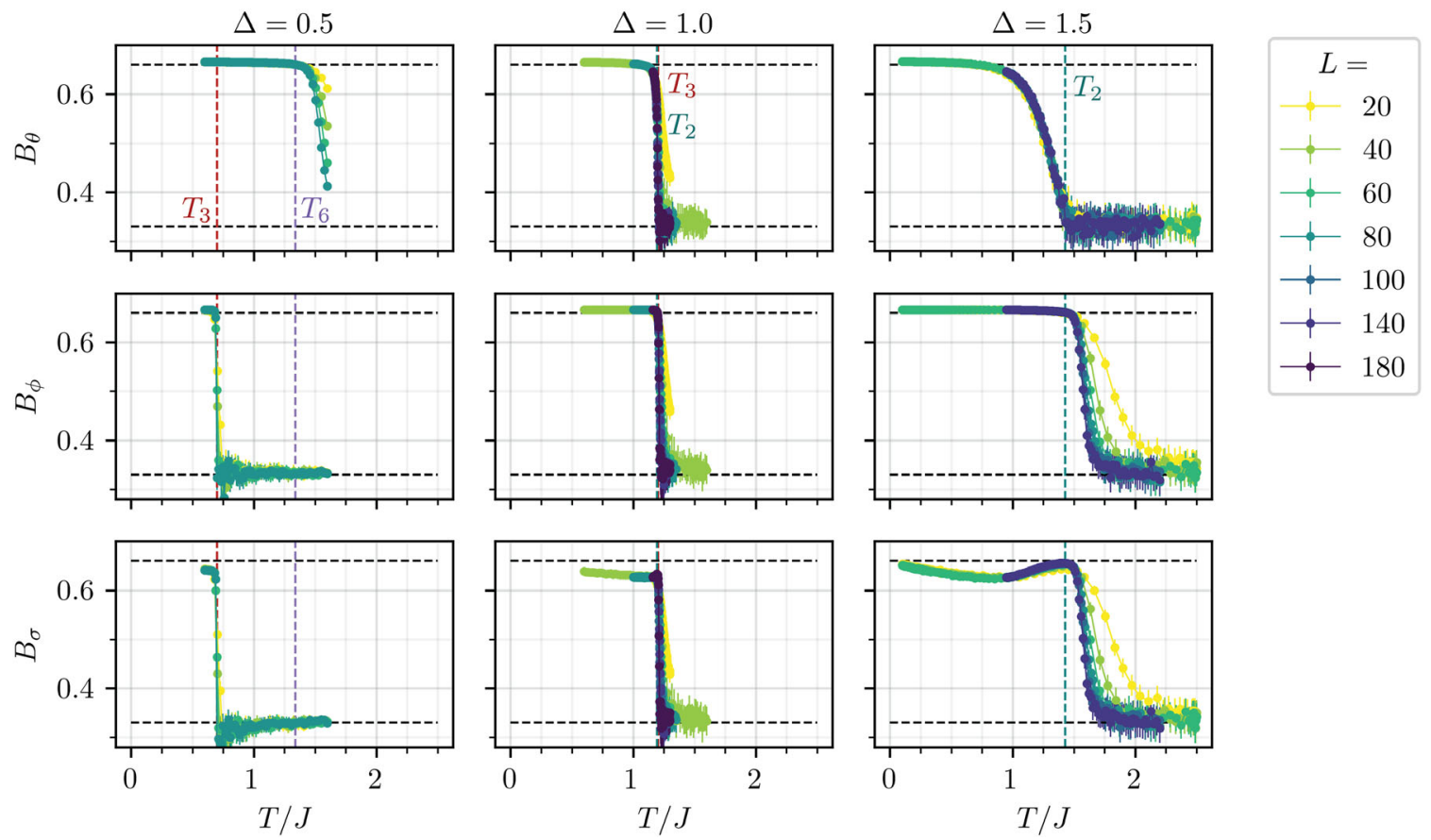

FIG. 10. Binder cumulants $B_{\theta}, B_{\phi}, B_{\sigma}$ of hexatic $\theta$, nematic $\phi$, and relative $\sigma=(\theta-3 \phi) / 3$ degrees of freedom as a function of the temperature $T / J$. Different columns denote different values of $\Delta=0.5,1.0,1.5$ as indicated. Vertical lines indicate the location of transition temperatures $T_{3}$ (Potts), $T_{6}$ (KT hexatic), and $T_{2}$ (KT nematic). 
temperature. In the $\Delta=0.5$ column, one observes that hexatic and Potts phase transitions are clearly separated with $T_{6}>T_{3}$, as signaled by the rapid increase of the respective Binder cumulants. At the Potts transition, both nematic and Potts Binder cumulants rapidly increase, and the system is fully (algebraically) ordered below $T_{3}$. Note that the increase of $B_{\theta}$ is significantly more broadened than that of $B_{\phi}$ and $B_{\sigma}$ as expected for a KT transition. In contrast, at $\Delta=1.5$, we observe that the nematic and Potts Binder cumulants increase at a temperature higher than the hexatic one. The transition is broad showing that this is a KT transition. Once the nematic degree of freedom is ordered, it induces a potential for the hexatic, which then tends to align with the nematic as the temperature is lowered, similar to a spin in a magnetic field. Correspondingly, the increase of $B_{\theta}$ occurs over a rather broad temperature range, corresponding to a crossover into the fully ordered state. Finally, at $\Delta=1.0$ we observe that all three Binder cumulants sharply increase close to $T=1.2 \mathrm{~J}$, suggesting the presence of a Potts transition. The crossing $B_{\sigma}(L)$ yields a transition temperature $T_{3} \simeq 1.202$ that is consistent with our findings in Fig. 4. Note that the Binder cumulants do not allow us to easily address the question whether the nematic and Potts transitions are separated. To demonstrate this, we rather rely on a refined scaling analysis presented in Fig. 6 in the main text.

[1] J. M. Kosterlitz and D. J. Thouless, Ordering, Metastability and Phase Transitions in Two-Dimensional Systems, J. Phys. C 6, 1181 (1973).

[2] J. M. Kosterlitz, The Critical Properties of the TwoDimensional XY Model, J. Phys. C 7, 1046 (1974).

[3] J. M. Kosterlitz, Kosterlitz-Thouless Physics: A Review of Key Issues, Rep. Prog. Phys. 79, 026001 (2016).

[4] E. Granato, J. M. Kosterlitz, and J. Poulter, Critical Behavior of Coupled XY Models, Phys. Rev. B 33, 4767 (1986).

[5] E. Granato, J. M. Kosterlitz, J. Lee, and M. P. Nightingale, Phase Transitions in Coupled XY-Ising Systems, Phys. Rev. Lett. 66, 1090 (1991).

[6] L. Radzihovsky, P. B. Weichman, and J. I. Park, Superfluidity and Phase Transitions in a Resonant Bose Gas, Ann. Phys. (Amsterdam) 323, 2376 (2008).

[7] L. de Forges de Parny, A. Rançon, and T. Roscilde, Multiple Transitions of Coupled Atom-Molecule Bosonic Mixtures in Two Dimensions, Phys. Rev. A 93, 023639 (2016).

[8] E. Babaev, Phase Diagram of Planar $U(1) \times U(1)$ Superconductor, Nucl. Phys. B686, 397 (2004).

[9] R. Hlubina, Possible Vortex Splitting in High-Temperature Cuprate Superconductors, Phys. Rev. B 77, 094503 (2008).

[10] Y. H. Kwan, G. Wagner, T. Soejima, M. P. Zaletel, S. H. Simon, S. A. Parameswaran, and N. Bultinck, Kekulé Spiral Order at All Nonzero Integer Fillings in Twisted Bilayer Graphene, Phys. Rev. X 11, 041063 (2021).
[11] R. Bruinsma and G. Aeppli, Hexatic Order and HerringBone Packing in Liquid Crystals, Phys. Rev. Lett. 48, 1625 (1982).

[12] G. Aeppli and R. Bruinsma, Hexatic Order and Liquid Density Fluctuations, Phys. Rev. Lett. 53, 2133 (1984).

[13] M. Gingras, P. Holdsworth, and B. Bergersen, Monte Carlo Study of Bond and Molecular Orientational Ordering in Two-Dimensional Nematic Liquid-Crystal Systems, Europhys. Lett. 9, 539 (1989).

[14] D. H. Lee and G. Grinstein, Strings in Two-Dimensional Classical XY Models, Phys. Rev. Lett. 55, 541 (1985).

[15] S. Korshunov, Possible Splitting of a Phase Transition in a 2D XY Model, JETP Lett. 41, 263 (1985).

[16] S.E. Korshunov, Phase Diagram of the Modified XY Model, J. Phys. C 19, 4427 (1986).

[17] P. Chaikin and T. C. Lubensky, Principles of Condensed Matter Physics (Cambridge University Press, Cambridge, England, 1995).

[18] L. Niu, S. Jin, X. Chen, X. Li, and X. Zhou, Observation of a Dynamical Sliding Phase Superfluid with P-Band Bosons, Phys. Rev. Lett. 121, 265301 (2018).

[19] S. Jin, W. Zhang, X. Guo, X. Chen, X. Zhou, and X. Li, Evidence of Potts-Nematic Superfluidity in a Hexagonal $s p^{2}$ Optical Lattice, Phys. Rev. Lett. 126, 035301 (2021).

[20] P. Chandra, P. Coleman, and A. I. Larkin, Ising Transition in Frustrated Heisenberg Models, Phys. Rev. Lett. 64, 88 (1990).

[21] C. Weber, L. Capriotti, G. Misguich, F. Becca, M. Elhajal, and F. Mila, Ising Transition Driven by Frustration in a $2 D$ Classical Model with Continuous Symmetry, Phys. Rev. Lett. 91, 177202 (2003).

[22] L. Capriotti, A. Fubini, T. Roscilde, and V. Tognetti, Ising Transition in the Two-Dimensional Quantum $J_{1}-J_{2}$ Heisenberg Model, Phys. Rev. Lett. 92, 157202 (2004).

[23] C. S. O'Hern and T. C. Lubensky, Sliding Columnar Phase of DNA-Lipid Complexes, Phys. Rev. Lett. 80, 4345 (1998).

[24] C. S. O’Hern, T. C. Lubensky, and J. Toner, Sliding Phases in XY Models, Crystals, and Cationic Lipid-DNA Complexes, Phys. Rev. Lett. 83, 2745 (1999).

[25] E. Fradkin and S. A. Kivelson, Liquid-Crystal Phases of Quantum Hall Systems, Phys. Rev. B 59, 8065 (1999).

[26] S. A. Kivelson, E. Fradkin, and V. J. Emery, Electronic Liquid-Crystal Phases of a Doped Mott Insulator, Nature (London) 393, 550 (1998).

[27] V. J. Emery, E. Fradkin, S. A. Kivelson, and T. C. Lubensky, Quantum Theory of the Smectic Metal State in Stripe Phases, Phys. Rev. Lett. 85, 2160 (2000).

[28] E. Fradkin, S. A. Kivelson, M. J. Lawler, J. P. Eisenstein, and A. P. Mackenzie, Nematic Fermi Fluids in Condensed Matter Physics, Annu. Rev. Condens. Matter Phys. 1, 153 (2010).

[29] R. M. Fernandes, P. P. Orth, and J. Schmalian, Intertwined Vestigial Order in Quantum Materials: Nematicity and Beyond, Annu. Rev. Condens. Matter Phys. 10, 133 (2019).

[30] A. J. Jin, M. Veum, T. Stoebe, C. F. Chou, J. T. Ho, S. W. Hui, V. Surendranath, and C.-C. Huang, Nature of the Smectic-A-Hexatic-B-Crystal-B Transitions of a Liquid-Crystal Compound, Phys. Rev. E 53, 3639 (1996). 
[31] C.-F. Chou, J. T. Ho, and S. W. Hui, Electron-Diffraction Study of a One-Layer Free-Standing Hexatic LiquidCrystal Film, Phys. Rev. E 56, 592 (1997).

[32] C.-F. Chou, A. J. Jin, S. Hui, C.-C. Huang, and J. T. Ho, Multiple-Step Melting in Two-Dimensional Hexatic Liquid-Crystal Films, Science 280, 1424 (1998).

[33] V. L. Berezinskii, Destruction of Long-Range Order in One-Dimensional and Two-Dimensional Systems Possessing a Continuous Symmetry Group, Sov. Phys. JETP 34, 610 (1972).

[34] I. M. Jiang, S. N. Huang, J. Y. Ko, T. Stoebe, A. J. Jin, and C. C. Huang, Monte Carlo Simulation of a Coupled XY Model, Phys. Rev. E 48, R3240 (1993).

[35] I. M. Jiang, T. Stoebe, and C. C. Huang, Monte Carlo Studies of Helicity Modulus and Heat Capacity of a Coupled XY Model in Two Dimensions, Phys. Rev. Lett. 76, 2910 (1996).

[36] A. F. Andreev and I. A. Grishchuk, Spin Nematics, Sov. Phys. JETP 60, 267 (1984).

[37] A. Nakatsuji, Y. Nambu, H. Tonomura, O. Sakai, S. Jonas, C. Broholm, H. Tsunetsugu, Y. Qiu, and Y. Maeno, Spin Disorder on a Triangular Lattice, Science 309, 1697 (2005).

[38] A. Mulder, R. Ganesh, L. Capriotti, and A. Paramekanti, Spiral Order by Disorder and Lattice Nematic Order in a Frustrated Heisenberg Antiferromagnet on the Honeycomb Lattice, Phys. Rev. B 81, 214419 (2010).

[39] M.E. Zhitomirsky, Octupolar Ordering of Classical Kagome Antiferromagnets in Two and Three Dimensions, Phys. Rev. B 78, 094423 (2008).

[40] C. L. Henley, The "Coulomb Phase” in Frustrated Systems, Annu. Rev. Condens. Matter Phys. 1, 179 (2010).

[41] P. P. Orth, P. Chandra, P. Coleman, and J. Schmalian, Emergent Critical Phase and Ricci Flow in a 2D Frustrated Heisenberg Model, Phys. Rev. Lett. 109, 237205 (2012).

[42] G.-W. Chern and R. Moessner, Dipolar Order by Disorder in the Classical Heisenberg Antiferromagnet on the Kagome Lattice, Phys. Rev. Lett. 110, 077201 (2013).

[43] H. D. Rosales, D. C. Cabra, C. A. Lamas, P. Pujol, and M.E. Zhitomirsky, Broken Discrete Symmetries in a Frustrated Honeycomb Antiferromagnet, Phys. Rev. B 87, 104402 (2013).

[44] P. P. Orth, P. Chandra, P. Coleman, and J. Schmalian, Emergent Criticality and Friedan Scaling in a TwoDimensional Frustrated Heisenberg Antiferromagnet, Phys. Rev. B 89, 094417 (2014).

[45] B. Jeevanesan, P. Chandra, P. Coleman, and P. P. Orth, Emergent Power-Law Phase in the 2D Heisenberg Windmill Antiferromagnet: A Computational Experiment, Phys. Rev. Lett. 115, 177201 (2015).

[46] C. Fang, H. Yao, W.-F. Tsai, J. P. Hu, and S. A. Kivelson, Theory of Electron Nematic Order in LaFeAsO, Phys. Rev. B 77, 224509 (2008).

[47] C. Xu, M. Müller, and S. Sachdev, Ising and Spin Orders in the Iron-Based Superconductors, Phys. Rev. B 78, 020501(R) (2008).

[48] R. M. Fernandes, L. H. VanBebber, S. Bhattacharya, P. Chandra, V. Keppens, D. Mandrus, M. A. McGuire,
B. C. Sales, A. S. Sefat, and J. Schmalian, Effects of Nematic Fluctuations on the Elastic Properties of Iron Arsenide Superconductors, Phys. Rev. Lett. 105, 157003 (2010).

[49] E. V. Herland, E. Babaev, and A. Sudbø, Phase Transitions in a Three Dimensional $U(1) \times U(1)$ Lattice London Superconductor: Metallic Superfluid and Charge-4e Superconducting States, Phys. Rev. B 82, 134511 (2010).

[50] J. W. F. Venderbos, L. Savary, J. Ruhman, P. A. Lee, and L. Fu, Pairing States of Spin-3/2 Fermions: SymmetryEnforced Topological Gap Functions, Phys. Rev. X 8, 011029 (2018).

[51] Y. Jiang, X. Lai, K. Watanabe, T. Taniguchi, K. Haule, J. Mao, and E. Y. Andrei, Charge Order and Broken Rotational Symmetry in Magic-Angle Twisted Bilayer Graphene, Nature (London) 573, 91 (2019).

[52] D. F. Agterberg, J. S. Davis, S. D. Edkins, E. Fradkin, D. J. Van Harlingen, S. A. Kivelson, P. A. Lee, L. Radzihovsky, J. M. Tranquada, and Y. Wang, The Physics of PairDensity Waves: Cuprate Superconductors and Beyond, Annu. Rev. Condens. Matter Phys. 11, 231 (2020).

[53] Y. Cao, D. Rodan-Legrain, J. M. Park, F. N. Yuan, K. Watanabe, T. Taniguchi, R. M. Fernandes, L. Fu, and P. Jarillo-Herrero, Nematicity and Competing Orders in Superconducting Magic-Angle Graphene, Science 372, 264 (2021).

[54] S. Gopalakrishnan, Y. E. Shchadilova, and E. Demler, Intertwined and Vestigial Order with Ultracold Atoms in Multiple Cavity Modes, Phys. Rev. A 96, 063828 (2017).

[55] T. C. Lubensky and H. Stark, Theory of a Critical Point in the Blue-Phase-III-Isotropic Phase Diagram, Phys. Rev. E 53, 714 (1996).

[56] D. R. Nelson and B. I. Halperin, Solid and Fluid Phases in Smectic Layers with Tilted Molecules, Phys. Rev. B 21, 5312 (1980).

[57] S. B. Dierker, R. Pindak, and R. B. Meyer, Consequences of Bond-Orientational Order on the Macroscopic Orientation Patterns of Thin Tilted Hexatic Liquid-Crystal Films, Phys. Rev. Lett. 56, 1819 (1986).

[58] J. M. Fellows, S. T. Carr, C. A. Hooley, and J. Schmalian, Unbinding of Giant Vortices in States of Competing Order, Phys. Rev. Lett. 109, 155703 (2012).

[59] M. Nitta, M. Eto, T. Fujimori, and K. Ohashi, Baryonic Bound State of Vortices in Multicomponent Superconductors, J. Phys. Soc. Jpn. 81, 084711 (2012).

[60] Y. Shi, A. Lamacraft, and P. Fendley, Boson Pairing and Unusual Criticality in a Generalized XY Model, Phys. Rev. Lett. 107, 240601 (2011).

[61] P. Serna, J. Chalker, and P. Fendley, Deconfinement Transitions in a Generalised XY Model, J. Phys. A 50, 424003 (2017).

[62] M. Kobayashi and M. Nitta, $Z_{n}$ Modified XY and Goldstone Models and Vortex Confinement Transition, Phys. Rev. D 101, 085003 (2020).

[63] M. Kobayashi, G. Fejős, C. Chatterjee, and M. Nitta, Vortex Confinement Transitions in the Modified Goldstone Model, Phys. Rev. Research 2, 013081 (2020).

[64] M. Kobayashi, M. Eto, and M. Nitta, Berezinskii-KosterlitzThouless Transition of Two-Component Bose Mixtures with 
Intercomponent Josephson Coupling, Phys. Rev. Lett. 123, 075303 (2019).

[65] A. M. Polyakov, Quark Confinement and Topology of Gauge Theories, Nucl. Phys. B120, 429 (1977).

[66] E. Fradkin and S. H. Shenker, Phase Diagrams of Lattice Gauge Theories with Higgs Fields, Phys. Rev. D 19, 3682 (1979).

[67] D. A. Tennant, T. G. Perring, R. A. Cowley, and S. E. Nagler, Unbound Spinons in the $S=1 / 2$ Antiferromagnetic Chain $\mathrm{KCuF}_{3}$, Phys. Rev. Lett. 70, 4003 (1993).

[68] M. Mourigal, M. Enderle, A. Klöpperpieper, J.-S. Caux, A. Stunault, and H. M. Rønnow, Fractional Spinon Excitations in the Quantum Heisenberg Antiferromagnetic Chain, Nat. Phys. 9, 435 (2013).

[69] A. Kitaev, Anyons in an Exactly Solved Model and Beyond, Ann. Phys. (Amsterdam) 321, 2 (2006).

[70] C. Castelnovo, R. Moessner, and S. L. Sondhi, Magnetic Monopoles in Spin Ice, Nature (London) 451, 42 (2008).

[71] R. B. Laughlin, Anomalous Quantum Hall Effect: An Incompressible Quantum Fluid with Fractionally Charged Excitations, Phys. Rev. Lett. 50, 1395 (1983).

[72] J. D. Brock, A. Aharony, R. J. Birgeneau, K. W. EvansLutterodt, J. D. Litster, P. M. Horn, G. B. Stephenson, and A. R. Tajbakhsh, Orientational and Positional Order in a Tilted Hexatic Liquid-Crystal Phase, Phys. Rev. Lett. 57, 98 (1986).

[73] M. Cheng, J. T. Ho, S. W. Hui, and R. Pindak, Observation of Two-Dimensional Hexatic Behavior in Free-Standing Liquid-Crystal Thin Films, Phys. Rev. Lett. 61, 550 (1988).

[74] K. J. Strandburg, Two-Dimensional Melting, Rev. Mod. Phys. 60, 161 (1988).

[75] P. Minnhagen, The Two-Dimensional Coulomb Gas, Vortex Unbinding, and Superfluid-Superconducting Films, Rev. Mod. Phys. 59, 1001 (1987).

[76] J. V. José, L. P. Kadanoff, S. Kirkpatrick, and D. R. Nelson, Renormalization, Vortices, and Symmetry-Breaking Perturbations in the Two-Dimensional Planar Model, Phys. Rev. B 16, 1217 (1977).

[77] B. I. Halperin and D. R. Nelson, Theory of TwoDimensional Melting, Phys. Rev. Lett. 41, 121 (1978).

[78] D. R. Nelson and B. I. Halperin, Dislocation-Mediated Melting in Two Dimensions, Phys. Rev. B 19, 2457 (1979).

[79] A. Young, Melting and the Vector Coulomb Gas in Two Dimensions, Phys. Rev. B 19, 1855 (1979).

[80] A. Levelut, Étude de l'ordre local lié a la rotation des molécules dans la phase smectique B, J. Phys. (Paris), Colloq. 37, C3-51 (1976).

[81] J. Doucet, Relation between the Herringbone Packing and the Chain Behaviour in the Ordered Smectic Phases, J. Phys. (Paris), Lett. 40, 185 (1979).

[82] M. J. P. Gingras, P. C. W. Holdsworth, and B. Bergersen, Induced Nearest-Neighbor Bond-Orientational Ordering and Director Fluctuations in Two-Dimensional LiquidCrystal Models, Phys. Rev. A 41, 3377 (1990).

[83] M. J. P. Gingras, P. C. W. Holdsworth, and B. Bergersen, Monte Carlo Study of Induced Bond Orientational Ordering in Two-Dimensional Liquid-Crystal Models, Phys. Rev. A 41, 6786 (1990).
[84] M. Gingras, B. Bergersen, and P. Holdsworth, Induced Nearest-Neighbor Bond Orientational Ordering and Structural Transformation in a Two-Dimensional Liquid Crystal Model, Mol. Cryst. Liq. Cryst. 204, 177 (1991).

[85] R. Pindak, D. E. Moncton, S. C. Davey, and J. W. Goodby, $X$-Ray Observation of a Stacked Hexatic Liquid-Crystal B Phase, Phys. Rev. Lett. 46, 1135 (1981).

[86] C.-F. Chou, J. T. Ho, S. W. Hui, and V. Surendranath, Scaling of 6n-Fold Bond-Orientational Order Parameters in a Hexatic Liquid-Crystal Thin Film, Phys. Rev. Lett. 76, 4556 (1996).

[87] T. Stoebe and C. Huang, Physical Properties of Thin Substrate-Free Liquid-Crystal Films, Int. J. Mod. Phys. B 09, 2285 (1995).

[88] M. Kohandel, M. J. P. Gingras, and J. P. Kemp, HexaticHerringbone Coupling at the Hexatic Transition in Smectic Liquid Crystals: $4-\varepsilon$ Renormalization Group Calculations Revisited, Phys. Rev. E 68, 041701 (2003).

[89] M. Hasenbusch, A. Pelissetto, and E. Vicari, Multicritical Behaviour in the Fully Frustrated XY Model and Related Systems, J. Stat. Mech. (2005) P12002.

[90] M. Hasenbusch, The Binder Cumulant at the KosterlitzThouless Transition, J. Stat. Mech. (2008) P08003.

[91] Y.-D. Hsieh, Y.-J. Kao, and A. W. Sandvik, Finite-Size Scaling Method for the Berezinskii-Kosterlitz-Thouless Transition, J. Stat. Mech. (2013) P09001.

[92] S. Romano, Topological Transitions in Two-Dimensional Lattice Spin Models, Phys. Rev. E 73, 042701 (2006).

[93] F. C. Poderoso, J. J. Arenzon, and Y. Levin, New Ordered Phases in a Class of Generalized XY Models, Phys. Rev. Lett. 106, 067202 (2011).

[94] G. A. Canova, Y. Levin, and J. J. Arenzon, KosterlitzThouless and Potts Transitions in a Generalized XY Model, Phys. Rev. E 89, 012126 (2014).

[95] G. A. Canova, Y. Levin, and J. J. Arenzon, Competing Nematic Interactions in a Generalized XY Model in Two and Three Dimensions, Phys. Rev. E 94, 032140 (2016).

[96] D. X. Nui, L. Tuan, N. D. T. Kien, P. T. Huy, H. T. Dang, and D. X. Viet, Correlation Length in a Generalized TwoDimensional XY Model, Phys. Rev. B 98, 144421 (2018).

[97] A. Roy, J. Hauschild, and F. Pollmann, Quantum Phases of a One-Dimensional Majorana-Bose-Hubbard Model, Phys. Rev. B 101, 075419 (2020).

[98] F.-F. Song and G.-M. Zhang, Hybrid BerezinskiiKosterlitz-Thouless and Ising Topological Phase Transition in the Generalized Two-Dimensional XY Model Using Tensor Networks, Phys. Rev. B 103, 024518 (2021).

[99] F.-Y. Wu, The Potts Model, Rev. Mod. Phys. 54, 235 (1982).

[100] R. Ghanbari and F. Shahbazi, Monte Carlo Simulation of a Strongly Coupled XY Model in Three Dimensions, Phys. Rev. E 72, 021709 (2005).

[101] L. de Forges de Parny and V. G. Rousseau, Quantum and Thermal Phase Transitions in a Bosonic Atom-Molecule Mixture in a Two-Dimensional Optical Lattice, Phys. Rev. A 95, 013606 (2017).

[102] F. Shahbazi and R. Ghanbari, Emergence of Hexatic and Threefold Hidden Order in Two-Dimensional Smectic Liquid Crystals: A Monte Carlo Study, Phys. Rev. E 74, 021705 (2006). 
[103] D. B. Carpenter and J. T. Chalker, The Phase Diagram of a Generalised XY Model, J. Phys. Condens. Matter 1, 4907 (1989).

[104] M. Žukovič, Multiple Phase Transitions in the XY Model with Nematic-like Couplings, Phys. Lett. A 382, 2618 (2018).

[105] L. Bonnes and S. Wessel, Half-Vortex Unbinding and Ising Transition in Constrained Superfluids, Phys. Rev. B 85, 094513 (2012).

[106] S. E. Korshunov, Phase Transitions in Two-Dimensional Systems with Continuous Degeneracy, Phys. Usp. 49, 225 (2006).

[107] T. C. Halsey, Topological Defects in the Fully Frustrated XY Model and in ${ }^{3} \mathrm{He}-A$ Films, J. Phys. C 18, 2437 (1985).

[108] M. Y. Choi and S. Doniach, Phase Transitions in Uniformly Frustrated XY Models, Phys. Rev. B 31, 4516 (1985).

[109] M. Yosefin and E. Domany, Phase Transitions in Fully Frustrated Spin Systems, Phys. Rev. B 32, 1778 (1985).

[110] S. Teitel and C. Jayaprakash, Phase Transitions in Frustrated Two-Dimensional XY Models, Phys. Rev. B 27, 598 (1983).

[111] J. Lee, J. M. Kosterlitz, and E. Granato, Monte Carlo Study of Frustrated XY Models on a Triangular and Square Lattice, Phys. Rev. B 43, 11531 (1991).

[112] P. Olsson, Two Phase Transitions in the Fully Frustrated XY Model, Phys. Rev. Lett. 75, 2758 (1995).

[113] G. S. Jeon, S. Y. Park, and M. Y. Choi, Double Transitions in the Fully Frustrated XY Model, Phys. Rev. B 55, 14088 (1997).

[114] P. Simon, The 2D $J_{1}-J_{2} X Y$ and $X Y$-Ising Models, Europhys. Lett. 39, 129 (1997).

[115] D. Loison and P. Simon, Monte Carlo Analysis of the Phase Transitions in the Two-Dimensional $J_{1}-J_{2} X Y$ Model, Phys. Rev. B 61, 6114 (2000).

[116] P. Olsson and S. Teitel, Kink-Antikink Unbinding Transition in the Two-Dimensional Fully Frustrated XY Model, Phys. Rev. B 71, 104423 (2005).

[117] L. Huijse, B. Bauer, and E. Berg, Emergent Supersymmetry at the Ising-Berezinskii-Kosterlitz-Thouless Multicritical Point, Phys. Rev. Lett. 114, 090404 (2015).

[118] All code, scripts, and data used in this work are included in a GitHub repository, https://github.com/orth-research/ potts-xy.

[119] E. Marinari and G. Parisi, Simulated Tempering: A New Monte Carlo Scheme, Europhys. Lett. 19, 451 (1992).

[120] K. Hukushima and K. Nemoto, Exchange Monte Carlo Method and Application to Spin Glass Simulations, J. Phys. Soc. Jpn. 65, 1604 (1996).

[121] U. Wolff, Collective Monte Carlo Updating for Spin Systems, Phys. Rev. Lett. 62, 361 (1989).
[122] B. Efron, The Jackknife, the Bootstrap, and Other Resampling Plans (Society for Industrial and Applied Mathematics, Philadelphia, USA, 1982), Vol. 38.

[123] K. Binder, Static and Dynamic Critical Phenomena of the Two-Dimensional q-State Potts Model, J. Stat. Phys. 24, 69 (1981).

[124] S. Martinos, A. Malakis, and I. Hadjiagapiou, Finite-Size Scaling Analysis of the Critical Behavior of the Baxter-Wu Model, Physica (Amsterdam) 352A, 447 (2005).

[125] I. N. Velonakis, Critical Energy Distribution Function of the Baxter-Wu Model, Physica (Amsterdam) 399A, 171 (2014).

[126] I. N. Velonakis, Efficient Energy Cumulants for the BaxterWu Model, Physica (Amsterdam) 422A, 153 (2015).

[127] M. E. Fisher, M. N. Barber, and D. Jasnow, Helicity Modulus, Superfluidity, and Scaling in Isotropic Systems, Phys. Rev. A 8, 1111 (1973).

[128] A. W. Sandvik, Computational Studies of Quantum Spin Systems, AIP Conf. Proc. 1297, 135 (2010).

[129] H. Weber and P. Minnhagen, Monte Carlo Determination of the Critical Temperature for the Two-Dimensional XY Model, Phys. Rev. B 37, 5986 (1988).

[130] D. M. Hübscher and S. Wessel, Stiffness Jump in the Generalized XY Model on the Square Lattice, Phys. Rev. E 87, 062112 (2013).

[131] K. Beach, L. Wang, and A. W. Sandvik, Data Collapse in the Critical Region Using Finite-Size Scaling with Subleading Corrections, arXiv:cond-mat/0505194.

[132] L. Wang, K. S. D. Beach, and A. W. Sandvik, HighPrecision Finite-Size Scaling Analysis of the QuantumCritical Point of $s=1 / 2$ Heisenberg Antiferromagnetic Bilayers, Phys. Rev. B 73, 014431 (2006).

[133] N. Prokof'ev and B. Svistunov, Worm Algorithms for Classical Statistical Models, Phys. Rev. Lett. 87, 160601 (2001).

[134] Y. Saito, Melting of Dislocation Vector Systems in Two Dimensions, Phys. Rev. Lett. 48, 1114 (1982).

[135] Y. Saito, Monte Carlo Studies of Two-Dimensional Melting: Dislocation Vector Systems, Phys. Rev. B 26, 6239 (1982).

[136] K. J. Strandburg, S. A. Solla, and G. V. Chester, Monte Carlo Studies of a Laplacian Roughening Model for TwoDimensional Melting, Phys. Rev. B 28, 2717 (1983).

[137] J. Iaconis, Analytical and Numerical Studies of $2 D X Y$ Models with Ring Exchange, Ph.D. thesis, University of Waterloo, 2012.

[138] L. P. Kadanoff, Scaling and Universality in Statistical Physics, Physica (Amsterdam) 163A, 1 (1990).

[139] J. Goryo, S. Soma, and H. Matsukawa, Deconfinement of Vortices with Continuously Variable Fractions of the Unit Flux Quanta in Two-Gap Superconductors, Europhys. Lett. 80, 17002 (2007). 